\title{
VECTOR CROSS PRODUCTS ON MANIFOLDS
}

\author{
BY \\ ALFRED GRAY $\left({ }^{1}\right)$
}

1. Introduction. Calabi [6] has shown that every orientable hypersurface of $R^{7}$ possesses an almost complex structure. The properties of these manifolds have been investigated by Calabi [6], Gray [11], and Yano and Sumitomo [22]. The almost complex structure, which is a generalization of the one on $S^{6}$, is defined in terms of a vector cross product on $R^{7}$, which is a generalization of the ordinary Gibbs vector cross product on $R^{3}$.

In this paper we give a general definition of vector cross product (\$2), and then study vector cross products on manifolds. Vector cross products are interesting for three reasons: first, they are themselves natural generalizations of the notion of almost complex structure; secondly, a vector cross product on a manifold $M$ gives rise to unusual almost complex structures on certain submanifolds of $M$; thirdly, vector cross products provide one approach to the study of Riemannian manifolds with holonomy group $G_{2}$ or Spin (7).

Vector cross products on vector spaces have been studied from an algebraic standpoint in Brown and Gray [5] and from a topological standpoint in Eckmann [9] and Whitehead [20].

We consider the topological existence of vector cross products on manifolds in $\S 2$. Then in $\$ 3$ we give conditions for the existence of vector cross products on vector bundles over $\mathrm{CW}$-complexes in terms of characteristic classes. As an extra feature of our investigations we determine the behavior of the triality automorphism of Spin (8) on Spin characteristic classes. In $\$ 4$ we discuss vector cross products from a differential geometric point of view and in $\$ 5$ we develop some important relations between vector cross products and curvatures.

The rest of the paper ( $\$ 6$ and $\S 7)$ is devoted to almost complex structures on manifolds. We show in $\S 6$ that every orientable 6-dimensional submanifold of $R^{8}$ possesses an almost complex structure. Such an almost complex structure is defined by means of a 3 -fold vector cross product on $R^{8}$. We also show that all of Calabi's manifolds can be obtained by this method.

However, the almost complex structures constructed here are more general in several ways. In the first place, we consider 6-dimensional orientable submanifolds $M$ of arbitrary 8-dimensional pseudo-Riemannian manifolds $\bar{M}$ possessing 3-fold vector cross products, e.g., parallelizable 8-dimensional manifolds. Secondly, the metric of $\bar{M}$ we use may be indefinite provided it has signature $(4,4)$. (Here we

Received by the editors October 3, 1967 and, in revised form, August 22, 1968.

( $\left.{ }^{1}\right)$ This work was partially supported by National Science Foundation grant GP-6974. 
follow [20] in the notion of signature. The first entry of the ordered pair denotes the number of -'s and the second entry the number of + 's.) This gives rise to some new indefinite 6-dimensional almost complex manifolds with interesting properties. Finally in both the positive definite and the indefinite case there exist two nonisomorphic 3-fold vector cross products-four altogether. Each of these gives rise to different almost complex structures; in particular we obtain some new almost complex structures on $S^{6}$. Furthermore in $\$ 6$ we construct manifolds with almost complex structures $J_{1}$ and $J_{2}$ defined by means of the two nonisomorphic vector cross products such that $J_{1}$ is Kählerian but $J_{2}$ is not.

After these almost complex manifolds have been defined, there remains the problem of relating the properties of the almost complex structures to the properties of the immersion. This has already been done (see [6], [11], and [22]) for immersions in $R^{7}$. In contrast to this case, an orientable 6-dimensional submanifold of $R^{8}$ which is Kählerian need not be flat, although in general it is noncompact. In general the configuration tensor (i.e., the second fundamental form, see below) of an almost complex manifold in $R^{8}$ is much more complicated than when the submanifold is contained in a hyperplane. Furthermore, it is not always clear geometrically what a given condition on the almost complex structure implies about the configuration tensor. The difficulty is that the geometry of hypersurfaces is well understood, but very little is known in general about the geometry of submanifolds of codimension 2. Perhaps the case we consider will give some insight into the general situation.

At any rate, our results yield new information (described in $\$ 7$ ) about almost complex manifolds in $R^{7}$ with either definite or indefinite metric, and the geometric interpretation is clear. Furthermore, in $\$ 7$ we describe explicit constructions of nonflat 6-dimensional Kähler manifolds in $R^{8}$.

We wish to thank David Handel for suggesting Theorem (2.8), Emery Thomas and Jerrold Siegel for several discussions concerning $\$ 3$, and Robert B. Brown for several general discussions about this paper.

\section{Vector cross products.}

DEFINITION. Let $V$ be a finite-dimensional vector space over the real numbers and let $\langle$,$\rangle be a nondegenerate bilinear form on V$. A vector cross product on $V$ is a multilinear map $P: V^{r} \rightarrow V(1 \leqq r \leqq n)$ satisfying the axioms

$$
\begin{gathered}
\left\langle P\left(a_{1}, \ldots, a_{r}\right), a_{i}\right\rangle=0 \quad(1 \leqq i \leqq r), \\
\left\|P\left(a_{1}, \ldots, a_{r}\right)\right\|^{2}=\operatorname{det}\left(\left\langle a_{i}, a_{j}\right\rangle\right) .
\end{gathered}
$$

(Here $\|a\|^{2}=\langle a, a\rangle$.)

Vector cross products were first defined by Eckmann [9]. However, he assumed they were continuous instead of multilinear and used only the positive definite bilinear form. In the continuous case vector cross products are classified in [9] and [20]; for the multilinear case (over an arbitrary field) the classification is given in [5]. 
Let $P$ and $P^{\prime}$ be two vector cross products on $V$ with respect to the same bilinear form. Suppose there is a map $\Phi: V \rightarrow V$ such that $\langle\Phi a, \Phi b\rangle=\langle a, b\rangle$ and

$$
P\left(\Phi a_{1}, \ldots, \Phi a_{r}\right)=(-1)^{q} P^{\prime}\left(a_{1}, \ldots, a_{r}\right) .
$$

If $q$ is even (odd) we say that $P$ and $P^{\prime}$ are isomorphic (anti-isomorphic).

We now describe the classification of multilinear vector cross products over the real numbers together with their automorphism and antiautomorphism groups. (The latter is the group of all automorphisms and antiautomorphisms.) There are four types.

Type I. 1-fold vector cross products on an even-dimensional vector space $V$. The bilinear form associated to any such vector cross products has signature $(2 p, 2 q)$. The automorphism group is $U(p, q)$ and the antiautomorphism group is a subgroup of $O(p, q)$ which is an extension of $U(p, q)$ by $Z_{2}$. Any two almost complex structures on $V$ are isomorphic, but not necessarily naturally; however, any almost complex structure $J$ is naturally anti-isomorphic to $-J$.

Type II. $(n-1)$-dimensional vector cross products on an $n$-dimensional space $V$. These are given by the star operators of the associated bilinear forms. Any bilinear form with signature $(2 p, n-2 p)$ gives rise to such a vector cross product. The automorphism groups are $S O(2 p, n-2 p)(0 \leqq 2 p \leqq n)$ and the antiautomorphism groups are $O(2 p, n-2 p)(0 \leqq 2 p \leqq n)$. For a given bilinear form there are exactly two $(n-1)$-fold vector cross products and these are naturally anti-isomorphic. They are also isomorphic but are not naturally isomorphic if $n$ is even.

Type III. 2-fold vector cross products on a 7-dimensional vector space $V$. These are described as follows. Let $W$ be a composition algebra and let $V$ be the orthogonal complement of the identity $e$. Define $P: V \times V \rightarrow V$ by $P(a, b)=a b+\langle a, b\rangle e$. Then $P$ is a 2 -fold vector cross product and conversely every such vector cross product arises in this fashion. If $\operatorname{dim} W=8$, we get a 2 -fold vector cross product on a 7-dimensional space. The bilinear form associated with such a vector cross product has signature $(0,7)$ or $(4,3)$. The automorphism group is either $G_{2}$ (compact form) or $G_{2}^{*}$ (noncompact form), and the antiautomorphism group is an extension of $G_{2}$ or $G_{2}^{*}$ by $Z_{2}$ and is a subgroup of either $O(7)$ or $O(4,3)$. Any two 2-fold vector cross products are isomorphic but not necessarily naturally; however, $P$ and $-P$ are naturally isomorphic (and anti-isomorphic).

Type IV. 3-fold vector cross products on an 8-dimensional vector space $V$. Let $V$ be a composition algebra and $\langle,\rangle_{1}$ the associated bilinear form. Define $P_{1}, P_{2}: V \times V \times V \rightarrow V$ by

$$
\begin{aligned}
& P_{1}(a, b, c)=\varepsilon\left(-a(\bar{b} c)+\langle a, b\rangle_{1} c+\langle b, c\rangle_{1} a-\langle c, a\rangle_{1} b\right) \\
& P_{2}(a, b, c)=\varepsilon\left(-(a \bar{b}) c+\langle a, b\rangle_{1} c+\langle b, c\rangle_{1} a-\langle c, a\rangle_{1} b\right)
\end{aligned}
$$

where $\varepsilon= \pm 1$. Then $P_{1}$ and $P_{2}$ are 3-fold vector cross products on $V$ with respect to the bilinear form $\varepsilon<,\rangle_{1}$, and conversely every 3-fold vector cross product 
arises in this fashion. If $\operatorname{dim} V=8$ we get two nonisomorphic 3-fold vector cross products with the same associated bilinear form. However, the conjugation in the composition algebra gives rise to an anti-isomorphism between $P_{1}$ and $P_{2}$. Every other 3-fold vector cross product with bilinear form $\varepsilon<, \quad\rangle_{1}$ is isomorphic to either $P_{1}$ or $P_{2}$. The bilinear form associated with 3 -fold vector cross products has signature $(0,8),(4,4)$, or $(8,0)$. The automorphism groups of both $P_{1}$ and $P_{2}$ are isomorphic to Spin (7) or Spin $(4,3)$, depending on the bilinear form. The two automorphism groups are conjugate in $O(8)$ or $O(4,4)$ via the conjugation in a composition algebra. Furthermore, the antiautomorphism group in each case is isomorphic to the automorphism group.

The fact that there are two 3-fold vector cross products on an 8-dimensional vector space which are anti-isomorphic, but not isomorphic, is equivalent to the following fact in group representations: there exist two representations of Spin (7) $($ Spin $(4,3))$ which are equivalent in $O(8)(O(4,4))$ but not in $S O(8)(S O(4,4))$.

We shall need the following result.

Proposition (2.1). Let $V$ be an 8-dimensional real vector space on which there exists a 3-fold vector cross product $P$. Let $\langle, \quad>$ be the bilinear form associated with $P$, and assume that $\langle, \quad>$ is positive definite or has signature $(4,4)$. Let $a, b \in V$ be such that $\|a\|^{2}=\|b\|^{2}=1$ and $\langle a, b\rangle=0$. Then, depending on the signature of the metric,

(i) the group $A_{1}$ of automorphisms of $P$ which fix $a$ and $b$ is isomorphic to $S U(3)$ or $S U(2,1)$;

(ii) the group $B_{2}$ of automorphisms of $P$ which leave invariant the subspace spanned by $a$ and $b$ is isomorphic to $U(3)$ or $U(2,1)$.

Proof. Let $W$ be the orthogonal complement of $a$ and $b$ in $V$. Define $J: W \rightarrow W$ by $J x=P(a, b, x)$, for $x \in W$. Then [5] $J$ is an almost complex structure on $W$ (see also Theorem (2.6)). The group $A_{1}$ is a connected Lie group and its Lie algebra $\mathfrak{A}_{1}$ is isomorphic to the derivations of $P$ (i.e., maps $D: V \rightarrow V$ such that $D P(x, y, z)$ $=P(D x, y, z)+P(x, D y, z)+P(x, y, D z))$ which annihilate $a$ and $b$. Hence each derivation in $\mathfrak{A}_{1}$ may be regarded as a map $D: W \rightarrow W$; then $D J=J D$. Let $\left\{e_{1}, J e_{1}, e_{2}, J e_{2}, e_{3}, J e_{3}\right\}$ be an orthogonal basis of $W$ with $\left\|e_{1}\right\|^{2}=\left\|e_{2}\right\|^{2}= \pm 1$, $\left\|e_{3}\right\|^{2}=1$, and $P\left(a, e_{1}, e_{2}\right)=e_{3}$. Then we may write

$$
D\left(e_{p}+(\sqrt{ }-1) J e_{p}\right)=\sum_{q=1}^{3} \theta_{p q}\left(e_{q}+(\sqrt{ }-1) J e_{q}\right)
$$

for $p=1,2,3$ : The matrix $\left(\theta_{p q}\right)$ is in the Lie algebra of $U(3)$ or $U(2,1)$. We show that the trace of $\left(\theta_{p q}\right)$ is 0 . We have

$$
(\sqrt{ }-1) \sum_{p=1}^{3} \theta_{p p}=\sum_{p=1}^{3}\left\langle D e_{p}, J e_{p}\right\rangle\left\|e_{p}\right\|^{2}
$$


Now

$$
\begin{aligned}
\left\langle D e_{3}, J e_{3}\right\rangle & =\left\langle D P\left(a, e_{1}, e_{2}\right), J P\left(a, e_{1}, e_{2}\right)\right\rangle \\
& =-\left\langle P\left(a, D e_{1}, e_{2}\right), P\left(a, J e_{1}, e_{2}\right)\right\rangle-\left\langle P\left(a, e_{1}, D e_{2}\right), P\left(a, e_{1}, J e_{2}\right)\right\rangle \\
& =-\left\langle D e_{1}, J e_{1}\right\rangle\left\|e_{1}\right\|^{2}-\left\langle D e_{2}, J e_{2}\right\rangle\left\|e_{2}\right\|^{2} .
\end{aligned}
$$

Hence $\left(\theta_{p q}\right)$ is in the Lie algebra of $S U(3)$ or $S U(2,1)$, and conversely any such matrix gives rise to an element of $\mathfrak{A}_{1}$. It follows that the Lie group $A$ is locally isomorphic to $S U(3)$ or $S U(2,1)$. In fact $A$ is globally isomorphic to $S U(3)$ or $S U(2,1)$ since it is a subgroup of $G_{2}$ or $G_{2}^{*}$. Finally (ii) may be proved in a similar manner.

In this paper we are interested in multilinear vector cross products on manifolds. First we investigate the existence of such. In other words, we ask the question: which (differentiable) manifolds have on each tangent space a vector cross product defined which varies continuously over the whole manifold? This question is reformulated by the following proposition, the proof of which is easy.

Proposition (2.2.) Let $M$ be a pseudo-Riemannian manifold with metric tensor $<, \quad>$ whose signature is $(s, t)$. Then $M$ has a globally defined vector cross product $P$ if and only if the principal bundle of frames with structure group $O(s, t)$ can be reduced to a principal bundle of frames whose structure group is the automorphism group of the vector cross product. The vector cross product is continuous or differentiable just as the reduction is continuous or differentiable.

From this we obtain the following consequence.

Proposition (2.3). A vector cross product on a differentiable manifold $M$ gives rise to an orientation of $M$.

Proof. It can be verified case by case that the automorphism group of a vector cross product is always contained in the special orthogonal group of the bilinear form of the vector cross product. Therefore, the reduction of the bundle of frames given by Proposition (2.2) implies a reduction of the bundle of frames to a bundle of oriented frames. This is equivalent to the orientability of $M$.

The question of existence of globally defined vector cross products of Types I and II has been extensively studied. Thus from Theorem (2.2) it follows that the existence of a global vector cross product of Type II is equivalent to the orientability of the pseudo-Riemannian manifold. Furthermore, the fact that on each tangent space there are precisely two vector cross products of Type II, and these are naturally anti-isomorphic, corresponds to the fact that the existence of one orientation implies the existence of the other. As noted previously, vector cross products of Type I which are globally defined give rise to almost complex manifolds. Since two almost complex structures on a vector space are not in general naturally isomorphic, it follows that a manifold may (and generally does) have more than one globally defined almost complex structure. 
Investigation of the "exceptional" vector cross products of Types III and IV is practically nonexistent. By Proposition (2.2) the existence of a global 2-fold vector cross product on a 7-dimensional manifold is equivalent to a reduction of the bundle of frames with group $O(7)$ or $O(4,3)$ to a $G_{2}$ or $G_{2}^{*}$ principal bundle. Similarly the existence of a global 3 -fold cross product on an 8-dimensional manifold is the same as a reduction of the bundle of frames with group $O(8)$ or $O(4,4)$ to a Spin $(7)$ or Spin $(4,3)$ principal bundle. Clearly every parallelizable manifold of dimension 7 or 8 has a 2 - or 3-fold vector cross product.

Another structure that an 8-dimensional manifold may possess is a Cayley multiplication.

Definitions. Let $M$ be an 8-dimensional pseudo-Riemannian manifold whose metric tensor $\langle,>$ is positive definite or has signature $(4,4)$. Then a Cayley multiplication on $M$ is a continuous or differentiable $(1,2)$ tensor field denoted by $\cdot$, which on each tangent space of $M$ gives rise to a composition algebra of dimension 8 whose associated bilinear form is $\langle$,$\rangle . The identity vector field E$ of the Cayley multiplication is that vector field which at each point $m \in M$ is the identity of the composition algebra at $m$. Finally, the conjugation of the Cayley multiplication is the map $\sigma: \mathfrak{X}(M) \rightarrow \mathfrak{X}(M)$ (where $\mathfrak{X}(M)$ denotes the vector fields of $M$ ) defined by $\sigma X=X-2\langle X, E\rangle E$ for $X \in \mathfrak{X}(M)$.

We remark that the existence of a Cayley multiplication on a manifold is equivalent to the reduction of the group of the bundle of frames of $M$ to $G_{2}$ or $G_{2}^{*}$.

For global 3-fold vector cross products certain other questions arise in addition to the question of existence. Suppose $M$ is an 8-dimensional manifold possessing one kind of 3-fold vector cross product. Does it possess the other kind? Also, is it possible to define a Cayley multiplication on $M$ ? We have the following result.

THEOREM (2.4). Let $M$ be an 8-dimensional pseudo-Riemannian manifold whose metric is either positive definite or has signature $(4,4)$. Consider the following conditions:

(A) $M$ has a globally defined vector field $E$ with $\|E\|^{2}=1$ everywhere.

(B) $M$ has one kind of 3-fold vector cross product.

(C) $M$ has both kinds of 3-fold vector cross products.

(D) $M$ has a Cayley multiplication.

Then $((\mathrm{A})$ and $(\mathrm{B})) \Rightarrow((\mathrm{C})$ and $(\mathrm{D})),(\mathrm{D}) \Rightarrow(\mathrm{C}) \Rightarrow(\mathrm{B})$, and $(\mathrm{D}) \Rightarrow(\mathrm{A})$.

Proof. For the first implication let $P_{1}$ be a 3-fold vector cross product on $M$. Define $\sigma: \mathfrak{X}(M) \rightarrow \mathfrak{X}(M)$ by $\sigma X=X-2\langle X, E\rangle E$ for $X \in \mathfrak{X}(M)$ and define $P_{2}$ by $P_{2}(X, Y, Z)=\sigma P(\sigma X, \sigma Y, \sigma Z)$. It is easily verified that $P_{2}$ is a 3 -fold vector cross product on $M$. Furthermore, $P_{2}$ is a vector cross product of the opposite kind to $P_{1}$, because [5] on each tangent space of $M, P$ and $P_{2}$ determine different kinds of vector cross products. 
To show that (A) and (B) imply the existence of a Cayley multiplication, for $A, B \in \mathfrak{X}(M)$ we define

$$
A \cdot B=-P_{1}(A, E, B)+\langle A, E\rangle B+\langle B, E\rangle A-\langle A, B\rangle E .
$$

Just as in [5] it follows that $\|A \cdot B\|^{2}=\|A\|^{2}\|B\|^{2}$. Hence $A \cdot B$ is a Cayley multiplication on $M$.

It is obvious that $(C) \Rightarrow(B)$, and $(D) \Rightarrow(C)$ follows from (2.3) and (2.4). To prove $(\mathrm{D}) \Rightarrow(\mathrm{A})$ we take $E$ to be the identity vector field of the Cayley multiplication.

We next describe three methods of constructing new vector cross products from old.

THEOREM (2.5). Let $M$ be a 6-dimensional pseudo-Riemannian manifold such that the structure group of the frame bundle of $M$ can be reduced to $S U(3)$ or $S U(2,1)$. Let $M^{\prime}$ be a 7-dimensional manifold and $\pi: M^{\prime} \rightarrow M$ a pseudo-Riemannian submersion (i.e., $\pi$ is onto and $\pi_{*}$ preserves lengths on $\left(\operatorname{ker} \pi_{*}\right)^{\perp}$ ) such that the metric of $M^{\prime}$ is positive definite on $\operatorname{ker} \pi_{*}$. Then $M^{\prime}$ has a 2 -fold vector cross product $P^{\prime}$.

Proof. The hypotheses imply the existence on $M$ of a 3-form $\lambda$ which can be described as the complex volume element of $M$. Let $m \in M^{\prime}$. Both $\lambda$ and the almost complex structure $J$ of $M$ give rise to tensors on $\left(\operatorname{ker} \pi_{*}\right)^{\perp}$, which we also denote by $\lambda$ and $J$. For $x, y, z \in M_{m}^{\prime}$ with $z \in \operatorname{ker} \pi_{*},\|z\|^{2}=1, x, y \in\left(\operatorname{ker} \pi_{*}\right)^{\perp}$, and $\langle y, x\rangle=\langle y, J x\rangle=0$, we define $P^{\prime}$ by $P^{\prime}(z, x)=J x . P^{\prime}(x, J x)=\|x\|^{2} z$, and $P^{\prime}(x, y)=w$, where $w \in\left(\operatorname{ker} \pi_{*}\right)^{\perp}$ is the unique element such that $\lambda(x, y, w)=1$. We require that $P^{\prime}$ be skew-symmetric, and use linearity to define $P^{\prime}$ on all of $M_{m}^{\prime}$. It can be verified that $P^{\prime}$ is a 2 -fold vector cross product on $M^{\prime}$.

THEOREM (2.6). Let $\bar{P}$ be a vector cross product with respect to a metric tensor $<, \quad>$ on a manifold $\bar{M}$. Let $M$ be an oriented submanifold of $\bar{M}$ such that the restrictions of $\langle, \quad>$ to $M$ and to the normal bundle of $M$ are nondegenerate and positive definite respectively. Then $\bar{P}$ induces a vector cross product $P$ on $M$ in a natural way.

Proof. Let $\operatorname{dim} \bar{M}-\operatorname{dim} M=k$ and suppose $\bar{P}$ is $r$-fold. Without loss of generality we may assume $r<k$. Define an $(r-k)$-fold vector cross product $P$ on $M$ by the formula

$$
P\left(A_{1}, \ldots, A_{r-k}\right)=\bar{P}\left(N_{1}, \ldots, N_{k}, A_{1}, \ldots, A_{r-k}\right)
$$

for $A_{1}, \ldots, A_{r-k} \in \mathfrak{X}(M)$. Here $N_{1}, \ldots, N_{k}$ are normal vector fields defined on an open subset of $M$ such that $\left\langle N_{i}, N_{j}\right\rangle=\delta_{i j}(1 \leqq i, j \leqq k)$ and $N_{1} \wedge \cdots \wedge N_{k}$ is consistent with the orientations of $M$ and $\bar{M}$ (the latter orientation is the one determined by $\bar{P}$ ). It is not hard to verify that the definition of $P$ is independent of the choice of $N_{1}, \ldots, N_{k}$ and that $P$ is in fact a vector cross product on $M$. 
Theorem (2.6) will be used in $\S 6$ to provide some new examples of 6-dimensional almost complex manifolds. Meanwhile we note the following consequence, which is a partial converse of Theorem (2.5).

THEOREM (2.7). Let $M$ be an oriented 6-dimensional submanifold of a 7-dimensional pseudo-Riemannian manifold $\bar{M}$ which possesses a 2-fold vector cross product $P$. Assume that the restrictions of the metric tensor $\langle,>$ of $\bar{M}$ to the tangent bundle and the normal bundle of $M$ are nondegenerate and positive definite respectively. Then the structure group of the bundle of frames of $M$ can be reduced to $S U(3)$ or $S U(2,1)$ (depending on whether or not the metric is positive definite).

Proof. The structure group $O(6)$ or $O(4,2)$ of the bundle $F(M)$ of orthonormal frames of $M$ can be reduced to the subgroup of the structure group $O(7)$ or $O(4,3)$ of the bundle $F(\bar{M})$ of orthonormal frames of $\bar{M}$ which leaves $P$ invariant and fixes the normal bundle of $M$. According to Proposition (2.1) this is $S U(3)$ or $S U(2,1)$.

THEOREM (2.8). Let $S^{n}$ denote the unit sphere in $R^{n+1}$ and let $\langle, \quad>$ denote the metric tensor of $S^{n}$ induced from the usual positive definite one on $R^{n+1}$. If $S^{n}$ has a globally defined $r$-fold vector cross product, then in the vector space sense there is an $(r+1)$-fold continuous vector cross product on $R^{n+1}$.

Proof. Let $X_{m}$ denote the $r$-fold vector cross product on $S^{n}$ at $m$. Define

$$
P:\left(R^{n+1}\right)^{r+1} \rightarrow R^{n+1}
$$

as follows. Let $a_{1}, \ldots, a_{r+1} \in R^{n+1}$ and write $a_{r+1}=b+c$, where $b$ is the component of $a_{r+1}$ normal to $a_{1}, \ldots, a_{r}$. If $b=0$ we set $P\left(a_{1}, \ldots, a_{r+1}\right)=0$. If $b \neq 0$, let $d=\|b\|^{-1} b$ and set

$$
P\left(a_{1}, \ldots, a_{r+1}\right)=\|b\| X_{d}\left(a_{1}, \ldots, a_{r}\right) .
$$

It is not hard to verify that $P$ is an $(r+1)$-fold vector cross product with respect to $\left\langle,>\right.$ on $R^{n+1}$. Note that $P$ is linear in $a_{1}, \ldots, a_{r}$, but in general it is only continuous in $a_{r+1}$.

Which spheres have vector cross products? The answer is easy for vector cross products of Types II and III. Since spheres are orientable they all have vector cross products of Type II; furthermore since $S^{7}$ is parallelizable it has a vector cross product of Type III. The existence of vector cross products of Type I on spheres is the well-known problem of the existence of almost complex structures on spheres. The problem can be solved very simply by means of Theorem (2.8).

COROLlaRY (2.9). The only spheres with almost complex structures are $S^{2}$ and $S^{6}$.

Proof. The existence of an almost complex structure on $S^{n}$ implies the existence of a 2 -fold continuous vector cross product on $R^{n+1}$. From [9] it follows that $n=2$ or 6 . 
Finally, concerning vector cross products of Type IV, we have the following result.

COROLlaRY (2.10). There does not exist a 3-fold vector cross product on $S^{8}$.

Proof. If $S^{8}$ had a 3 -fold vector cross product, then the vector space $R^{9}$ would have a continuous 4-fold vector cross product. However, by [20] this is impossible.

3. Obstructions to the existence of vector cross products. Let $M$ be a $\mathrm{CW}$ complex and $\xi=\left(E, M, p, R^{n}\right)$ a vector bundle. A vector cross product on $M$ is a continuous function which assigns to each point $q \in M$ a vector cross product on the fiber over $q$. In this section we give necessary and, for the most part, sufficient conditions in terms of characteristic classes that a vector bundle possess a vector cross product.

It is not hard to see that a necessary and sufficient condition for the existence of a vector cross product of Type I on $\xi$ is the vanishing of the Stiefel-Whitney class $w_{1}(\xi)$. For the existence of an almost complex structure on $\xi$, it is well known that the vanishing of the odd-dimensional Stiefel-Whitney classes is a necessary condition. Furthermore if $\operatorname{dim} M \leqq 8$ sufficient conditions are given by Massey [14], generalizing the work of Ehresmann and $\mathrm{Wu}$. Therefore we restrict ourselves to vector cross products of Types III and IV.

We first give some necessary conditions for the existence of vector cross products of types III and IV analogous to those of Types I and II described above. These conditions could be determined from the cohomology of the classifying spaces $B G_{2}$ and $B$ Spin (7) (see Borel [3]). However, we find it more natural to derive these conditions by means of obstruction theory. This is because Borel gives the cohomology of $B G_{2}$ and $B$ Spin (7) only with $Z_{2}$ as coefficient group, whereas some of the Stiefel-Whitney classes we consider are integral classes.

THEOREM (3.1). If $\xi=\left(E, M, p, R^{n}\right)(n=7$ or 8$)$ is a vector bundle which possesses a vector cross product of Type III or IV, then the Stiefel-Whitney classes $w_{1}(\xi) \in H^{1}\left(M, Z_{2}\right), w_{2}(\xi) \in H^{2}\left(M, Z_{2}\right), W_{3}(\xi) \in H^{3}(M, Z)$, and $W_{5}(\xi) \in H^{5}(M, Z)$ $\operatorname{vanish}\left({ }^{2}\right)$.

Proof. Let $\xi_{k}=\left(E_{k}, M, p_{k}, V_{n, n-k}\right)$ be the associated bundle of $\xi$ whose fiber is the Stiefel manifold $V_{n, n-k}$, let $M_{j}$ denote the $j$-skeleton of $M$, and let $E_{k} \mid M_{j}$ denote the restriction of $E_{k}$ to $M_{j}$. By definition $w_{k}(\xi) \in H^{k}\left(M, \pi_{k-1}\left(V_{n, n-k+1}\right)\right)$ is the obstruction to the existence of a cross section of $\xi_{k+1}$ over $M_{k}$. We show, for example, that $W_{5}(\xi)=0$ when $\xi$ has a vector cross product of Type III.

We know that $\xi_{5}=\left(E_{5}, M, p_{5}, V_{7,2}\right)$ has a cross section $f$ on $M_{5}$ because the first nonzero homotopy group of $V_{7,2}$ is $\pi_{5}\left(V_{7,2}\right)=Z_{2}$. Now a vector cross product

$\left({ }^{2}\right)$ We denote by $W_{i}$ an integral Stiefel-Whitney class and by $w_{i}$ either a mod 2 StiefelWhitney class or the reduction mod 2 of an integral Stiefel-Whitney class. 
of Type III gives rise to a cross section of the bundle $V_{7,3} \rightarrow V_{7,2}$, and hence to a cross section $g$ of the bundle $E_{4} \rightarrow E_{5}$. Thus we have

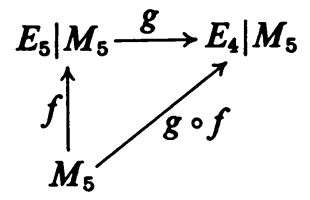

It follows that $W_{5}(\xi)=0$. The vanishing of the other Stiefel-Whitney classes follows in exactly the same way.

If $\operatorname{dim} M \leqq 7$, we can completely solve the question of existence of vector cross products of Types III and IV.

THEOREM (3.2). Let $\xi=\left(E, M, p, R^{n}\right)(n=7$ or 8$)$ be an oriented vector bundle. Then $w_{2}(\xi)$ is the primary obstruction to the existence on $M$ of a vector cross product of Type III (if $n=7)$ or Type IV (if $n=8$ ).

Proof. Observe that both $S O(7) / G_{2}$ and $S O(8) /$ Spin (7) are diffeomorphic to real projective space $P^{7}$. Also, $\xi$ possesses a vector cross product if and only if the associated bundle $\left(E, M, p, P^{7}\right)$ has a cross section. By naturality, it suffices to determine the primary obstruction $\bar{c}(\xi)$ to the existence of this cross section for the case $M=B G_{2}$ or $B$ Spin (7). The first and second cohomology groups of $B G_{2}$ and $B$ Spin (7) vanish. Therefore transgression $t$ yields isomorphisms

$$
t: H^{1}\left(P^{7}, Z_{2}\right) \cong H^{2}\left(B S O(7), Z_{2}\right)
$$

and

$$
t: H^{1}\left(P^{7}, Z_{2}\right) \cong H^{2}\left(B S O(8), Z_{2}\right) \text {. }
$$

The theorem now follows from the fact that

$$
t(\bar{c}(\xi))=w_{2}(\xi)
$$

(see Steenrod [16, p. 189]).

Corollary (3.3). Let $\xi=\left(E, M, p, R^{n}\right)(n=7$ or 8) be a vector bundle, and assume $\operatorname{dim} M \leqq 7$. If $w_{1}(\xi)=w_{2}(\xi)=0$, then $W_{3}(\xi)=W_{5}(\xi)=0$.

We next develop the theory of characteristic classes using the Lie groups $G_{2}$ and Spin (7). We define these characteristic classes by means of obstruction theory in the same way that the Chern and Stiefel-Whitney classes are defined.

We note that we have the following sequences of fiberings by spheres:

$$
G_{2} \stackrel{S^{3}}{\longrightarrow} G_{2} / S U(2) \stackrel{S^{5}}{\longrightarrow} G_{2} / S U(3)=S^{6} ;
$$

$$
\operatorname{Spin}(7) \stackrel{S^{3}}{\longrightarrow} \operatorname{Spin}(7) / S U(2) \stackrel{S^{5}}{\longrightarrow} \operatorname{Spin}(7) / S U(3) \stackrel{S^{6}}{\longrightarrow} \operatorname{Spin}(7) / G_{2}=S^{7} \text {. }
$$

Define $G^{0}=G_{2}, G^{4}=G_{2} / S U(2), G^{6}=G_{2} / S U(3), H^{0}=\operatorname{Spin}(7), H^{4}=\operatorname{Spin}(7) / S U(2)$, $H^{6}=\operatorname{Spin}(7) / S U(3)$, and $H^{7}=\operatorname{Spin}(7) / G_{2}$. Now let ${ }^{G} \mathscr{B}$ and ${ }^{H} \mathscr{B}$ be $S^{6}$ - and $S^{7}$ - 
bundles over a CW-complex $M$. Define ${ }^{G} \mathscr{B}^{q}$ and ${ }^{H} \mathscr{B}^{a}$ to be the associated bundles of ${ }^{G} \mathscr{B}$ and ${ }^{H} \mathscr{B}$ with fiber $G^{q}$ and $H^{q}$ respectively. If ${ }^{G} \mathscr{B}^{q}$ and ${ }^{H} \mathscr{B}^{q}$ denote the respective total spaces, we have the following sequences of bundles

$$
\begin{aligned}
& { }^{G} B^{0} \rightarrow{ }^{G} B^{4} \rightarrow{ }^{G} B^{6} \rightarrow M \\
& { }^{H} B^{0} \rightarrow{ }^{H} B^{4} \rightarrow{ }^{H} B^{6} \rightarrow{ }^{H} B^{7} \rightarrow M .
\end{aligned}
$$

(Compare Steenrod [16, p. 190 and p. 210].) Now if $\mathscr{B}$ is any bundle we denote by $\bar{c}(\mathscr{B})$ the primary obstruction of $\mathscr{B}$.

Definition. If ${ }^{G} \mathscr{B}$ and ${ }^{H} \mathscr{B}$ are $S^{6}$ and $S^{7}$ bundles over a CW-complex $M$, we set

$$
\begin{aligned}
& c_{G}^{4}\left({ }^{(} \mathscr{B}\right)=\bar{c}\left({ }^{G} \mathscr{B}^{0}\right) \in H^{4}\left(M, \pi_{3}\left(G_{2}\right)\right)=H^{4}(M, Z) \\
& c_{G}^{6}\left({ }^{G} \mathscr{B}\right)=\bar{c}\left({ }^{G} \mathscr{B}^{4}\right) \in H^{6}\left(M, \pi_{5}\left(G_{2} / S U(2)\right)\right)=H^{6}\left(M, Z_{2}\right) \\
& c_{G}^{7}\left({ }^{G} \mathscr{B}\right)=\bar{c}\left({ }^{G} \mathscr{B}^{6}\right) \in H^{7}\left(M, \pi_{6}\left(S^{6}\right)\right)=H^{7}(M, Z) \\
& c_{H}^{4}\left({ }^{H} \mathscr{B}\right)=\bar{c}\left({ }^{H} \mathscr{B}^{\circ}\right) \in H^{4}\left(M, \pi_{3}(\operatorname{Spin}(7))\right)=H^{4}(M, Z) \\
& c_{H}^{6}\left({ }^{H} \mathscr{B}\right)=\bar{c}\left({ }^{H} \mathscr{B}^{4}\right) \in H^{6}\left(M, \pi_{5}(\operatorname{Spin}(7) / S U(2))\right)=H^{6}\left(M, Z_{2}\right) \\
& c_{H}^{7}\left({ }^{H} \mathscr{B}\right)=\bar{c}\left({ }^{H} \mathscr{B}^{6}\right) \in H^{7}\left(M, \pi_{6}(\operatorname{Spin}(7) / S U(3))\right)=H^{7}(M, Z) \\
& c_{H}^{8}\left({ }^{H} \mathscr{B}\right)=\bar{c}\left({ }^{H} \mathscr{B}^{7}\right) \in H^{8}\left(M, \pi_{7}\left(S^{7}\right)\right)=H^{8}(M, Z) .
\end{aligned}
$$

Just as for the Chern and Stiefel-Whitney classes, we have the following theorem.

TheOREM (3.4). The classes $c_{G}^{4}\left({ }^{G} \mathscr{B}\right), c_{G}^{6}\left({ }^{G} \mathscr{B}\right)$, and $c_{G}^{7}\left({ }^{G} \mathscr{B}\right)$ are the characteristic classes of the bundle ${ }^{G} \mathscr{B}$ with respect to the Lie group $G_{2}$, and the classes $c_{H}^{4}\left({ }^{H} \mathscr{B}\right)$, $\ldots, c_{H}^{8}\left({ }^{H} \mathscr{B}\right)$ are the characteristic classes of the bundle ${ }^{H} \mathscr{B}$. Furthermore if $g: G_{2}$ $\rightarrow S O(7)$ and $j:$ Spin $(7) \rightarrow S O(8)$ denote the inclusions we have $g^{*}\left(c_{G}^{7}\left({ }^{G} \mathscr{B}\right)\right)=W^{7}\left({ }^{G} \mathscr{B}\right)$, $j^{*}\left(c_{H}^{7}\left({ }^{H} \mathscr{B}\right)\right)=W^{7}\left({ }^{H} \mathscr{B}\right)$, and $\left.j^{*}\left(c_{H}^{8}{ }^{H} \mathscr{B}\right)\right)=W^{8}\left({ }^{H} \mathscr{B}\right)$, where the $W^{i}$ s denote integral Stiefel-Whitney classes.

Corollary (3.5). We have $H^{*}\left(B G_{2}, Z_{2}\right)=Z_{2}\left[t^{4}, t^{6}, t^{7}\right]$ and $H^{*}\left(B\right.$ Spin (7), $\left.Z_{2}\right)$, $=Z_{2}\left[t^{4}, t^{6}, t^{7}, t^{8}\right]$.

It will be useful to have more information about the generators of

$$
H^{*}\left(B \text { Spin (7), } Z_{2}\right. \text { ), }
$$

and also of $H^{*}\left(B\right.$ Spin (8), $\left.Z_{2}\right)$. Let $q$ : Spin (8) $\rightarrow S O(8)$ denote the covering homomorphism, and let $q^{*}: H^{*}\left(B S O(8), Z_{2}\right) \rightarrow H^{*}\left(B\right.$ Spin (8), $\left.Z_{2}\right)$ be the induced cohomology map of $q$. Write $H^{*}\left(B S O(8), Z_{2}\right)=Z_{2}\left[w_{2}, \ldots, w_{8}\right]$, where the $w_{i}$ 's are the universal Stiefel-Whitney classes. The following theorem is an immediate consequence of Borel [4]. (See also Thomas [18].)

THEOREM (3.6). We have $q^{*} w_{2}=q^{*} w_{3}=q^{*} w_{5}=0$. For $i=4,6,7,8$ set $w_{i}^{*}=q^{*} w_{i}$. Then

$$
H^{*}\left(B \operatorname{Spin}(7), Z_{2}\right)=Z_{2}\left[w_{4}^{*}, w_{6}^{*}, w_{7}^{*}, \omega\right]
$$

and

$$
H^{*}\left(B \operatorname{Spin}(8), Z_{2}\right)=Z_{2}\left[w_{4}^{*}, w_{6}^{*}, w_{7}^{*}, w_{8}^{*}, \omega\right],
$$

where $\omega$ is an additional 8-dimensional generator. 
We call $\omega$ the exceptional Spin characteristic class. It is only determined mod $w_{8}^{*}$ and $w_{4}^{* 2}$; however we shall later make a specific choice.

Let $\xi=\left(E, M, p, R^{8}\right)$ be an oriented vector bundle with $\operatorname{dim} M \geqq 8$. Since $S O(8) /$ Spin (7) $=P^{7}$, the secondary obstruction to the existence of a 3-fold vector cross product on $\xi$ is an element of $H^{8}\left(M, \pi_{7}\left(P^{7}\right)\right)=H^{8}(M, Z)$. We conclude this section by determining some necessary conditions for this obstruction to vanish. To this end let $i:$ Spin (7) $\rightarrow$ Spin (8) and $j:$ Spin (7) $\rightarrow S O(8)$ denote the inclusions, and let $\lambda$ : Spin (8) $\rightarrow$ Spin (8) be the triality automorphism. The last is an outer automorphism of Spin (8) of order 3 with fixed point set $G_{2}$.

Proposition (3.7). Let $P_{1}$ and $P_{2}$ be the 3-fold vector cross products given by (2.3) and (2.4), respectively, with respect to an 8-dimensional composition algebra $V$, and let $\mathrm{H}_{1}$ and $\mathrm{H}_{2}$ be the corresponding automorphism groups. For $A \in \mathrm{Spin}$ (8) and $x \in V$ let $A x=q(A) x$. Then

$$
\begin{aligned}
& H_{1}=\{q(A) \mid A \in \text { Spin (8) and } A(x y)=(A x)(\lambda(A) y) \text { for all } x, y \in V\}, \\
& H_{2}=\{q(A) \mid A \in \text { Spin (8) and } A(x y)=(\lambda(A) x)(A y) \text { for all } x, y \in V\} .
\end{aligned}
$$

We omit the proof, which follows from the principle of triality and a calculation. An important consequence of Proposition (3.7) is the following. (Compare [19].)

Proposition (3.8). We have the following commutative diagram.

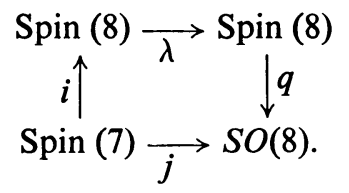

Next we turn to the cohomology homomorphisms $j^{*}$ and $\lambda^{*}$ on $Z_{2}$ cohomology.

THEOREM (3.9). (i) We have $j^{*}\left(w_{i}\right)=w_{i}^{*}$ for $i=4,6,7$.

(ii) $\omega$ can be chosen so that $j^{*}\left(w_{8}\right)=\omega$.

(iii) We have $\lambda^{*}\left(w_{i}\right)=w_{i}^{*}$ for $i=4,6,7$.

(iv) $\omega$ can be chosen so that $\lambda^{*}\left(w_{8}\right)=\omega$.

Proof. (iii) follows from the fact that $\lambda^{*}$ is bijective and $H^{i}\left(B \operatorname{Spin}(8), Z_{2}\right)$ is 1-dimensional for $i=4,6,7$. Hence (i) also follows. From Theorem (3.4) we have that $j^{*}\left(w_{8}\right) \neq 0$; this implies (ii) and (iv).

Henceforth we agree that $\omega$ is chosen so that $\lambda^{*}\left(w_{8}\right)=\omega$; this implies automatically that $j^{*}\left(w_{8}\right)=\omega$.

We turn now to the cohomology homomorphisms $j^{*}$ and $\lambda^{*}$ on integral cohomology. For this it is convenient to use the integral Spin characteristic classes of Thomas [17]. According to [17] there exist $Q_{1}, Q_{2}, Q_{3}$ in $H^{*}(B$ Spin (8), $Z$ ) (or in $H^{*}(B$ Spin $\left.(7), Z)\right)$ such that $Z\left[Q_{1}, Q_{2}, Q_{3}\right]$ is free and $q^{*}\left(P_{1}\right)=Q_{1}, q^{*}\left(P_{2}\right)=2 Q_{2}$ $+Q_{1}^{2}, q^{*}\left(P_{3}\right)=Q_{3}$, where $P_{1}, P_{2}, P_{3} \in H^{*}(B S O(8), Z)$ denote Pontrjagin classes. 
THeOREM (3.10). (i) $\lambda^{*}$ is the identity on $Z\left[Q_{1}, Q_{2}, Q_{3}\right]$.

(ii) We have $j^{*}\left(P_{1}\right)=2 Q_{1}, j^{*}\left(P_{2}\right)=2 Q_{2}+Q_{1}^{2}$, and $j^{*}\left(P_{3}\right)=Q_{3}$.

(iii) If $\chi \in H^{*}(B S O(8), Z)$ denotes the Euler class and $\rho_{2}$ denotes reduction $\bmod 2$, then $\rho_{2} j^{*}(\chi)=\omega$ and $2 j^{*}(\chi)=0$.

Proof. Let $T$ be a maximal torus of Spin (8) such that $\lambda(T)=T$. It is not hard to see that $\lambda^{*}$ is the identity on $H^{*}(B T, Z)$. This implies (i). Then (ii) follows from (i) and Proposition (3.8). For (iii) we first observe that $\rho_{2} j^{*}(\chi)=j^{*}\left(\rho_{2} \chi\right)=j^{*}\left(w_{8}\right)=\omega$. Next let $\rho_{R}$ denote the homomorphism from integral cohomology to real cohomology. Then $\rho_{R} j^{*}(\chi)=0$ and so $2 j^{*}(\chi)=0$.

Using standard properties of universal bundles, we obtain immediately from Theorem (3.10) some necessary conditions for the existence of 3-fold vector cross products. Denote by $\chi(\xi), w_{8}(\xi)$, and $\omega(\xi)$ the Euler class, the eighth StiefelWhitney class, and the exceptional Spin characteristic class of a vector bundle $\xi$. (The last is defined if $\xi$ has a 3-fold vector cross product.)

THEOREM (3.11). Let $\xi=\left(E, M, p, R^{8}\right)$ be an oriented vector bundle, and assume that $\xi$ has a 3-fold vector cross product. Then $2 \chi(\xi)=0$ and $w_{8}(\xi)=\omega(\xi)$.

COROLlaRy (3.12). Let $M$ be an 8-dimensional manifold and assume the tangent bundle $\tau(M)$ of $M$ has a continuous 3-fold vector cross product on it. Then $\chi(\tau(M))$ $=\omega(\tau(M))=0$.

Proof. Since $2 \chi(\tau(M))=0, \chi(\tau(M))=0$.

We now see that the nonexistence of a 3 -fold vector cross product on $S^{8}$ is a special case of Corollary (3.12).

4. Differentiable vector cross products. In this section we investigate vector cross products from the viewpoint of Riemannian geometry. Let $M$ be a differentiable manifold equipped with a pseudo-Riemannian metric $\langle, \quad\rangle$, and as before let $\mathfrak{X}(M)$ denote the Lie algebra of vector fields on $M$. We denote by $\nabla$ the Riemannian connection of $M$. If $P$ is an $r$-fold differentiable (i.e., $C^{\infty}$ ) vector cross product on $M$, then $P$ is a tensor field on $M$ of type $(r, 1)$. From the definition it is evident that every such vector cross product $P$ determines a global differential form $\varphi$ of degree $r+1$ by the formula

$$
\varphi\left(X_{1}, \ldots, X_{r+1}\right)=\left\langle P\left(X_{1}, \ldots, X_{r}\right), X_{r+1}\right\rangle
$$

for $X_{1}, \ldots, X_{r+1} \in \mathfrak{X}(M)$. Furthermore we have

$$
\nabla_{z}(\varphi)\left(X_{1}, \ldots, X_{r+1}\right)=\left\langle\nabla_{z}(P)\left(X_{1}, \ldots, X_{r}\right), X_{r+1}\right\rangle
$$

for $Z, X_{1}, \ldots, X_{r+1} \in \mathfrak{X}(M)$. A natural question is: which manifolds possess parallel vector cross products (i.e., $\nabla_{Z}(P)=0$ for $\left.Z \in \mathfrak{X}(M)\right)$ ? The answer is provided by the following theorem. 
THEOREM (4.1). A pseudo-Riemannian manifold $M$ has a parallel vector cross product $P$ if and only if at each point $m \in M$ the holonomy group of $M$ at $m$ is a subgroup of the automorphism group of $P$ at $m$.

Proof. We regard the holonomy group $\mathscr{H}_{m}$ at $m \in M$ as the group of nonsingular linear maps $\tau_{y}: M_{m} \rightarrow M_{m}$ which are parallel translations along (piecewise differentiable) closed curves $\gamma$ starting at $m$.

Now suppose $P$ is a vector cross product on $M$. Then the condition $\nabla_{Z}(P)=0$ for all $Z \in \mathfrak{X}(M)$ is equivalent to the statement that if $\gamma$ is a closed curve starting at $m$ and $X_{1}, \ldots, X_{r}$ are vector fields parallel along $\gamma$, then $P\left(X_{1}, \ldots, X_{r}\right)$ is also parallel along $\gamma$. This is equivalent to saying that each $\tau_{\gamma}$ is an automorphism of $P$. Hence Theorem (4.1) follows.

Berger [1] has classified all possible candidates for holonomy groups of manifolds having affine connections with zero torsion. These include all the automorphism groups of vector cross products. We shall see that if a pseudo-Riemannian manifold has a vector cross product $P$ of Type II, i.e., if the manifold is orientable, then $P$ is parallel. The existence of a parallel vector cross product of Type I means that the pseudo-Riemannian manifold is Kählerian.

If a pseudo-Riemannian manifold has a parallel vector cross product of Type III or IV, then the holonomy group in the positive definite case is contained in $G_{2}$ or Spin (7) and in the indefinite case is contained in $G_{2}^{*}$ or Spin $(4,3)$. Recently Bonan [2] has investigated compact Riemannian manifolds whose holonomy groups are $G_{2}$ or Spin (7). He has shown that in the former case such manifolds have nonzero Betti numbers $b_{3}$ and $b_{4}$ and in the latter case such manifolds have nonzero Betti number $b_{4}$. In fact it follows from (4.2) that $\varphi$ is a parallel differential form and hence generates the cohomology in dimensions 3 or 4 of Riemannian manifolds with holonomy groups $G_{2}$ or Spin (7). Bonan has also shown that the cohomology of these manifolds has other properties corresponding to the various relations between the Betti numbers of Kähler manifolds.

However, it turns out [2] that the Ricci curvature of a Riemannian manifold with holonomy group $G_{2}$ or Spin (7) vanishes. For this reason it is unclear that any Riemannian manifolds with these holonomy groups exist. On the other hand, we shall show that there exist many interesting examples of pseudo-Riemannian manifolds with vector cross products of Types I, III, or IV which are not parallel, but satisfy some slightly weaker conditions.

We shall consider classes of triples $(M, P,\langle, \quad\rangle)$ where $P$ is a vector cross product on a pseudo-Riemannian manifold $M$ with metric tensor $\langle,>$ such that $\langle, \quad\rangle$ is associated to $P$. Such a class is determined by the vector cross product $P$, since by definition a vector cross product has a bilinear form associated with it. Therefore we suppress $M$ and $\langle,>$ from the notation, but these are always understood to be given by the vector cross product $P$.

Definitions. Let $M$ be a pseudo-Riemannian manifold with metric tensor 
$\langle, \quad>$ and suppose $P$ is an $r$-fold differentiable vector cross product on $M$ associated with $\langle$,$\rangle . Let \nabla$ and $\delta$ denote the Riemannian connection and coderivative, respectively, of $M$ relative to $\langle\quad, \quad\rangle$, and let $\varphi$ denote the $(r+1)$-fold differential form determined by $P$.

(i) $P$ is nearly parallel if $\nabla_{X_{1}}(P)\left(X_{1}, X_{2}, \ldots, X_{r}\right)=0$ for all $X_{1}, \ldots, X_{r} \in \mathfrak{X}(M)$.

(ii) $P$ is almost parallel if $d \varphi=0$.

(iii) $P$ is semiparallel if $\delta \varphi=0$.

We denote by $\mathscr{P}(s, t, r), \mathscr{N} \mathscr{P}(s, t, r), \mathscr{A} \mathscr{P}(s, t, r)$, and $\mathscr{S} \mathscr{P}(s, t, r)$ the classes of $r$-fold vector cross products on pseudo-Riemannian manifolds whose metric tensors have signature $(s, t)$ which are parallel, nearly parallel, almost parallel, and semiparallel respectively. Sometimes we write $\mathscr{P}$ instead of $\mathscr{P}(s, t, r)$, etc.

These definitions, together with two additional ones given in [11] (namely the classes $\mathscr{2} \mathscr{K}$ and $\mathscr{H}$ of quasi-Kählerian and Hermitian manifolds) which do not generalize to general vector cross products, have been given for almost complex manifolds in [11]. We sometimes follow the notation of [11] and write " $\mathscr{K}$ " for " $\mathscr{P}$ " and replace "parallel" by "Kähler". For example, we write $\mathscr{P}(s, t, 1)$ $=\mathscr{K}(s, t)$, and similarly for the other classes.

In [11] and [13] it is shown that an almost complex structure $J$ is Hermitian if and only if it is integrable.

We establish some inclusion relations between the various classes.

THEOREM (4.2). We have the following inclusions:

(i) $\mathscr{P} \subseteq \mathscr{N} \mathscr{P} \subseteq \mathscr{S} \mathscr{P}$;

(ii) $\mathscr{P} \subseteq \mathscr{A} \mathscr{P}$;

(iii) $\mathscr{P}=\mathscr{A} \mathscr{P} \cap \mathscr{N} \mathscr{P}$.

Proof. The inclusion $\mathscr{P} \subseteq \mathscr{N} \mathscr{P}$ is obvious from the definitions and the inclusions $\mathscr{N} \mathscr{P} \subseteq \mathscr{S} \mathscr{P}$ and $\mathscr{P} \subseteq \mathscr{A} \mathscr{P}$ follow from the equations

$$
\begin{gathered}
\delta \varphi\left(X_{1}, \ldots, X_{r}\right)=-\sum_{i=1}^{n}\left\|E_{i}\right\|^{-2} \nabla_{E_{i}}(\varphi)\left(E_{i}, X_{1}, \ldots, X_{r}\right) \\
d \varphi\left(X_{1}, \ldots, X_{r+2}\right)=\sum_{i=1}^{r+2}(-1)^{i+1} \nabla_{X_{i}}(\varphi)\left(X_{1}, \ldots, \hat{X}_{i}, \ldots, X_{r+2}\right)
\end{gathered}
$$

where $X_{1}, \ldots, X_{r+2} \in \mathfrak{X}(M)$ and $\left\{E_{1}, \ldots, E_{n}\right\}$ is an orthogonal frame field with $\left\|E_{i}\right\|^{2}= \pm 1$. This proves (i) and (ii). Then (iii) follows from the formula

$$
d \varphi\left(X_{1}, \ldots, X_{r+2}\right)=(r+2) \nabla_{X_{1}}(\varphi)\left(X_{2}, \ldots, X_{r+2}\right)
$$

which is valid for the class $\mathscr{N} \mathscr{P}$.

Next we look at each of the different types of vector cross products individually to determine further inclusion relations. First, however, we note a useful formula.

Proposition (4.3). Let $X, X_{1}, \ldots, X_{r} \in \mathfrak{X}(M)$. Then

$$
\left\langle\nabla_{X}(P)\left(X_{1}, \ldots, X_{r}\right), P\left(X_{1}, \ldots, X_{r}\right)\right\rangle=0 .
$$


Let $\mathscr{A} \mathscr{H}$ denote the class of all almost complex structures with $\langle J X, J Y\rangle$ $=\langle X, Y\rangle$ for $X, Y \in \mathfrak{X}(M)$.

THEOREM (4.4). For almost complex structures (i.e., vector cross products of Type I) the following inclusions hold:

(i) $\mathscr{K} \subseteq \mathscr{N} \mathscr{K} \subseteq \mathscr{Q} \mathscr{K} \subseteq \mathscr{S} \mathscr{K} ; \mathscr{K} \subseteq \mathscr{A} \mathscr{K} \subseteq \mathscr{Q} \mathscr{K} \subseteq \mathscr{S} \mathscr{K}$;

(ii) $\mathscr{K} \subseteq \mathscr{H}$;

(iii) $\mathscr{K}=\mathscr{A} \mathscr{K} \cap \mathscr{N} \mathscr{K}=\mathscr{H} \cap \mathscr{Q} \mathscr{K}$;

(iv) $\mathscr{K}(0,2)=\mathscr{A} \mathscr{H}(0,2)$;

(v) If $s$ and $t$ are even and $s+t=4$, then $\mathscr{K}(s, t)=\mathscr{N} \mathscr{K}(s, t)$ and $\mathscr{A} \mathscr{K}(s, t)$ $=\mathscr{S} \mathscr{K}(s, t)$.

Proof. (i), (ii), and (iii) are established in [10] and [11] for the positive definite case; the proofs go over without change to the indefinite case. Furthermore (iv) is well known.

To prove (v) let $F$ denote the associated 2-form of $J$ (called the Kähler form). We note that if $J \in \mathscr{Q} \mathscr{K}$ then

$$
d F(J X, Y, Z)=d F(X, J Y, Z)=d F(X, Y, J Z)
$$

for all $X, Y, Z \in \mathfrak{X}(M)$.

Now suppose that $M$ is 4-dimensional. We may choose an orthogonal basis for the vector fields on an open subset of $M$ to be of the form $\{X, J X, Y, J Y\}$ where $\|X\|^{2} \neq 0 \neq\|Y\|^{2}$. If $J \in \mathscr{Q} \mathscr{K}(s, t), s+t=4$, then by (4.6) $d F(X, J X, Y)=$ $d F(Y, J Y, X)=0$. Hence $\mathscr{A} \mathscr{K}(s, t)=\mathscr{Q} \mathscr{K}(s, t)$. If $J \in \mathscr{S} \mathscr{K}(s, t)$, then by Proposition (4.3) and equation (4.3)

$$
\delta F(X)=-\nabla_{Y}(F)(Y, X)-\nabla_{J Y}(F)(J Y, X)
$$

and similarly for $J X, Y$, and $J Y$. Thus $J \in \mathscr{Q} \mathscr{K}(s, t)$, and so $\mathscr{S} \mathscr{K}(s, t)=\mathscr{Q} \mathscr{K}(s, t)$ $=\mathscr{A} \mathscr{K}(s, t)$. This fact together with (i) and (iii) implies that $\mathscr{K}(s, t)=\mathscr{N} \mathscr{K}(s, t)$.

Proposition (4.5). A vector cross product $P$ of Type II on a manifold is always parallel.

This follows from the fact that the associated differential form of $P$ is the volume element of $M$.

Finally we consider vector cross products of Type IV.

TheOREM (4.6). We have $\mathscr{P}(0,8,3)=\mathscr{N} \mathscr{P}(0,8,3), \mathscr{P}(4,4,3)=\mathscr{N} \mathscr{P}(4,4,3)$, and $\mathscr{P}(8,0,3)=\mathscr{N} \mathscr{P}(8,0,3)$.

Proof. Let $P$ be a 3 -fold vector cross product on an 8-dimensional pseudoRiemannian manifold. It suffices to show that if $P$ is nearly parallel, then it is parallel. Consider $\nabla_{\mathrm{W}}(P)(X, Y, Z)$ where $W, X, Y, Z \in \mathfrak{X}(M)$ are nonisotropic. If $Z=\lambda P(W, X, Y)$, then for any $U \in \mathfrak{X}(M)$ we have

$$
\left\langle\nabla_{W}(P)(X, Y, Z), U\right\rangle=\lambda\left\langle\nabla_{U}(P)(W, X, Y), P(W, X, Y)\right\rangle=0
$$


by Proposition (4.3). Thus we may assume that $W, X, Y, Z$ and $P(W, X, Y)$ are orthogonal. Then on an open subset of $M,\{W, X, Y, Z, P(W, X, Y), P(W, X, Z)$, $P(W, Y, Z), P(X, Y, Z)\}$ forms an orthogonal basis for each tangent space. Then by Proposition (4.3), $\nabla_{W}(P)(X, Y, Z)$ is orthogonal to each of the vectors $W, X, Y, Z, P(W, X, Y), P(W, X, Z), P(W, Y, Z)$ and $P(X, Y, Z)$, and so it vanishes. Finally, the case when some of the vectors $W, X, Y, Z$ are isotropic can be reduced to the nonisotropic case by linearity.

Next we give two methods for constructing vector cross products. These will be useful in proving the strictness of the various inclusion relations.

Let $M$ be a submanifold of a pseudo-Riemannian manifold $\bar{M}$ such that the restriction of the metric tensor $\langle,>$ of $\bar{M}$ to $M$ is nondegenerate. Let $\overline{\mathfrak{X}}(M)$ $=\{X|M| X \in \mathfrak{X}(\bar{M})\}$; then we may write $\overline{\mathfrak{X}}(M)=\mathfrak{X}(M) \oplus \mathfrak{X}(M)^{\perp}$ where $\mathfrak{X}(M)^{\perp}$ is the collection of vector fields normal to $M$. The configuration tensor $T: \mathfrak{X}(M)$ $\times \overline{\mathfrak{X}}(M) \rightarrow \overline{\mathfrak{X}}(M)$ is defined by $T_{X} Y=\bar{\nabla}_{X} Y-\nabla_{X} Y$ for $X, Y \in \mathfrak{X}(M)$ and $T_{X} Z$ $=\pi \bar{\nabla}_{X} Z$ for $X \in \mathfrak{X}(M), Z \in \mathfrak{X}(M)^{\perp}$. Here $\bar{\nabla}$ and $\nabla$ are the Riemannian connections of $M$ and $\bar{M}$ respectively and $\pi$ is the projection of $\overline{\mathfrak{X}}(M)$ onto $\mathfrak{X}(M)$. Then [10] for each $X \in \mathfrak{X}(M), T_{X}$ is a skew-symmetric linear operator with respect to $\langle\quad, \quad\rangle$, and $T_{X} Y=T_{Y} X$ for $X, Y \in \mathfrak{X}(M)$.

Proposition (4.7). Let $M$ and $\bar{M}$ be pseudo-Riemannian manifolds with $\operatorname{dim} \bar{M}$ $-\operatorname{dim} M=k$ which satisfy the hypotheses of Theorem (2.6). Let the vector cross product $\bar{P}$ on $\bar{M}$ be r-fold so that the vector cross product $P$ on $M$ is $(r-k)$-fold. Then for $X, X_{1}, \ldots, \dot{X}_{\mathrm{r}-k} \in \mathfrak{X}(M)$ we have

$$
\begin{aligned}
\nabla_{X}(P)\left(X_{1}, \ldots, X_{r-k}\right)= & \pi \bar{\nabla}_{X}(\bar{P})\left(N_{1}, \ldots, N_{k}, X_{1}, \ldots, X_{r-k}\right) \\
& +\sum_{j=1}^{k} \pi \bar{P}\left(N_{1}, \ldots, T_{X} N_{j}, \ldots, N_{k}, X_{1}, \ldots, X_{r-k}\right),
\end{aligned}
$$

where $N_{1}, \ldots, N_{k}$ are the same as in the proof of Theorem (2.6). Hence if $\bar{P}$ is parallel and $M$ is totally geodesic in $\bar{M}$, then $P$ is parallel.

Proof. Note that in each term of the sum on the right-hand side of (4.7) $T_{A} N_{j}$ may be replaced by $\bar{\nabla}_{A} N_{j}$ because the normal component of $\bar{\nabla}_{A} N_{j}$ is orthogonal to $N_{j}$. Now (4.7) follows from the definition of $\bar{\nabla}_{A}(\bar{P})$.

We now derive several consequences of Theorem (4.7). We denote by $\varphi$ and $\bar{\varphi}$ the differential forms associated with the vector cross products $P$ and $\bar{P}$ of $M$ and $\bar{M}$ respectively.

THEOREM (4.8). Let $M$ and $\bar{M}$ be pseudo-Riemannian manifolds with $\operatorname{dim} \bar{M}$ $-\operatorname{dim} M=1$ which satisfy the hypotheses of Theorem (4.7). Assume that $\bar{P} \in \mathscr{N} \mathscr{P}(s, t, r)$. If $M$ is totally umbilic in $\bar{M}$, then $P \in \mathscr{N P}(s, t-1, r-1)$. The converse is also true if $s+t=7, r=2$, or if $s+t=8, t \geqq 4, r=3$.

Proof. By definition $M$ is totally umbilic in $\bar{M}$ if and only if there exists a differentiable real-valued function $\kappa$ on $M$ (depending on the unit normal $N$ to $M$ ) 
such that $T_{X} N=\kappa X$ for all $X \in \mathfrak{X}(M)$. Hence if $M$ is totally umbilic in $\bar{M}$, (4.7) reduces to

$$
\begin{aligned}
\nabla_{X}(P)\left(X_{1}, \ldots, X_{r-k}\right)= & \pi \bar{\nabla}_{X}(\bar{P})\left(N_{1}, \ldots, N_{k}, X_{1}, \ldots, X_{r-k}\right) \\
& +\kappa \sum_{j=1}^{k} \pi \bar{P}\left(N_{1}, \ldots, X, \ldots, N_{k}, X_{1}, \ldots, X_{r-k}\right) .
\end{aligned}
$$

It follows easily from this equation that if $\bar{P}$ is nearly parallel, so is $P$.

To prove the converse first suppose $s+t=7, r=2$, and write $P=J$, an almost complex structure. Then if $\bar{\nabla}_{X}(\bar{P})(X)=\nabla_{X}(J)(X)=0$ for all $X \in \mathfrak{X}(M)$, we have $\left\langle\bar{P}\left(T_{X} N, X\right), Y\right\rangle=0$ for all $X, Y \in \mathfrak{X}(M)$. It follows that $T_{X} N$ is a linear combination of $X$ and $J X$. Finally $\left\langle T_{X} N, J X\right\rangle=0$ because $J$ is quasi-Kählerian.

Suppose that $s+t=8$ and $r=3$. If $\bar{\nabla}_{X}(\bar{P})(X)=\nabla_{X}(P)(X)=0$ for all $X \in \mathfrak{X}(M)$, then $\left\langle\bar{P}(X, Y, Z), T_{X} N\right\rangle=0$ for all $X, Y, Z \in \mathfrak{X}(M)$. Upon writing $\bar{P}(X, Y, Z)$ out at each point in terms of a Cayley multiplication and (2.3) or (2.4), it is not hard to see that any vector field $W$ linearly independent from $X$ can be written as $W=\bar{P}(X, Y, Z)$ for some vector fields $Y$ and $Z$ in $\mathfrak{X}(M)$. It follows that $T_{X} N=\kappa X$ and so $M$ is totally umbilic.

Suppose that $M$ is an orientable hypersurface of $\bar{M}$ and that the metric of $M$ is positive definite. If $N$ is a unit normal to $M$ then the map $X \rightarrow T_{X} N$ (usually called the second fundamental form) is a symmetric linear operator. We denote by $\kappa_{1}, \ldots, \kappa_{n}(n=\operatorname{dim} M)$ the eigenvalues of $X \rightarrow T_{X} N$.

TheOREM (4.9). Let $M$ and $\bar{M}$ be Riemannian manifolds with $\operatorname{dim} \bar{M}-\operatorname{dim} M=1$ which satisfy the hypotheses of Theorem (4.7). Assume $\bar{P} \in \mathscr{P}(0,8,3)$. If $P \in \mathscr{A} \mathscr{P}(0,7,2)$, then $P$ is parallel and $M$ is totally geodesic in $\bar{M}$.

Proof. We show that $T$ vanishes at each point $m \in M$. Choose vector fields $X_{1}$ $X_{2}$ such that at $m$ we have $T_{X_{i}} N=\kappa_{i} X_{i}, i=1,2$. Let $Z \in \mathfrak{X}(M)$; then at $m$ by (4.7) we have

$$
\begin{aligned}
0 & =d \varphi\left(X_{1}, X_{2}, P\left(X_{1}, X_{2}\right), Z\right) \\
& =-\left\langle\bar{P}\left(X_{1}, X_{2}, Z\right),\left(\kappa_{1}+\kappa_{2}\right)\left(P\left(X_{1}, X_{2}\right)+T_{P\left(X_{1}, X_{2}\right)} N\right)\right\rangle .
\end{aligned}
$$

Since $Z$ is arbitrary and since we already have that $T_{P\left(X_{1}, X_{2}\right)} N$ is perpendicular to $X_{1}$ and $X_{2}$, it follows that we may write $T_{P\left(X_{1}, X_{2}\right)} N=\kappa_{4} P\left(X_{1}, X_{2}\right)$ at $m$. (Here $\kappa_{4}=-\kappa_{1}-\kappa_{2}$, but we do not use this fact.) Now choose $X_{3} \in \mathfrak{X}(M)$ orthogonal to $X_{1}, X_{2}$ and $P\left(X_{1}, X_{2}\right)$ such that $T_{X_{3}} N=\kappa_{3} X_{3}$ at $m$. Just as before we may write $T_{P\left(X_{1}, X_{3}\right)} N=\kappa_{5} P\left(X_{1}, X_{3}\right)$ and $T_{P\left(X_{2}, X_{3}\right)} N=\kappa_{6} P\left(X_{2}, X_{3}\right)$ at $m$. Using (4.7) again, we find that for any $Z \in \mathfrak{X}(M)$,

$$
0=d \varphi\left(X_{1}, X_{2}, X_{3}, Z\right)=\left(\kappa_{1}+\kappa_{2}+\kappa_{3}\right)\left\langle\bar{P}\left(X_{1}, X_{2}, X_{3}\right), Z\right\rangle+\left\langle T_{Z} N, \bar{P}\left(X_{1}, X_{2}, X_{3}\right)\right\rangle \text {. }
$$

Hence at $m$ we may write $T_{\bar{P}\left(X_{1}, X_{2}, X_{3}\right)} N=\kappa_{7} \bar{P}\left(X_{1}, X_{2}, X_{3}\right)$, where $\kappa_{7}=-\left(\kappa_{1}+\kappa_{2}+\kappa_{3}\right)$. We write $X_{4}=P\left(X_{1}, X_{2}\right), X_{5}=P\left(X_{1}, X_{3}\right), X_{6}=P\left(X_{2}, X_{3}\right), X_{7}=\bar{P}\left(X_{1}, X_{2}, X_{3}\right)$; then 
$\left\{X_{1}, \ldots, X_{7}\right\}$ is a basis for the vector fields in a neighborhood of $m$ such that $T_{X_{i}} N=\kappa_{i} X_{i}, i=1, \ldots, 7$, at $m$. We permute the $X_{i}$ 's; then using (2.3) or (2.4) at $m$ and repeating the process above, we obtain the following equations:

$$
\begin{aligned}
& \kappa_{1}+\kappa_{2}+\kappa_{3}+\kappa_{7}=0 \\
& \kappa_{1}+\kappa_{2}+\kappa_{5}+\kappa_{6}=0 \\
& \kappa_{1}+\kappa_{3}+\kappa_{4}+\kappa_{6}=0 \\
& \kappa_{1}+\kappa_{4}+\kappa_{5}+\kappa_{7}=0 \\
& \kappa_{2}+\kappa_{3}+\kappa_{4}+\kappa_{5}=0 \\
& \kappa_{2}+\kappa_{4}+\kappa_{6}+\kappa_{7}=0 \\
& \kappa_{3}+\kappa_{5}+\kappa_{6}+\kappa_{7}=0 .
\end{aligned}
$$

It is not hard to verify that the unique solution to this system is $\kappa_{1}=\cdots=\kappa_{7}=0$.

Hence $T$ vanishes at $m$; since $m$ is arbitrary, $M$ is totally geodesic in $\bar{M}$. From (4.7) it follows also that $P$ is parallel.

Let $\delta$ and $\delta$ denote the coderivatives of $M$ and $\bar{M}$ respectively; also, we denote by $\iota_{X}(X \in \mathfrak{X}(M))$ the interior product operator. (If $\theta$ is a $p$-form, $\iota_{X}(\theta)\left(X_{1}, \ldots, X_{p-1}\right)$ $=\theta\left(X, X_{1}, \ldots, X_{p-1}\right)$ for vector fields $X_{1}, \ldots, X_{p-1}$.)

TheOREM (4.10). Let $M$ and $\bar{M}$ be pseudo-Riemannian manifolds with $\operatorname{dim} \bar{M}$ $-\operatorname{dim} M=1$ which satisfy the hypotheses of Theorem (4.7). Then we have

$$
\delta \varphi=-\iota_{N}(\delta \bar{\varphi})
$$

where $N$ is the unit normal of $M$ in $\bar{M}$. Hence if $P \in \mathscr{S} \mathscr{P}(s, t, r)$, then

$$
P \in \mathscr{S P}(s, t-1, r-1) \text {. }
$$

Proof. If $\operatorname{dim} \bar{M}=7$, the proof of (4.8) is given in [11]. (In the indefinite case a few sign changes occur.) Therefore we give the proof of (4.8) for $\operatorname{dim} \bar{M}=8$.

Let $\left\{X_{1}, \ldots, X_{7}\right\}$ be an orthogonal basis on an open subset of $M$ with $X_{4}=$ $P\left(X_{1}, X_{2}\right), \quad X_{5}=P\left(X_{1}, X_{3}\right), \quad X_{6}=P\left(X_{2}, X_{3}\right)$, and $X_{7}=\bar{P}\left(X_{1}, X_{2}, X_{3}\right)$. Then by Proposition (4.3) and Proposition (4.7) we have

$$
\begin{aligned}
\delta \varphi\left(X_{1}, X_{2}\right)+\delta \bar{\varphi}\left(N, X_{1}, X_{2}\right) & \\
= & -\left\|X_{3}\right\|^{-2} \bar{\varphi}\left(T_{X_{3}} N, X_{3}, X_{1}, X_{2}\right)-\left\|X_{5}\right\|^{-2} \bar{\varphi}\left(T_{X_{5}} N, X_{5}, X_{1}, X_{2}\right) \\
& -\left\|X_{6}\right\|^{-2} \bar{\varphi}\left(T_{X_{6}} N, X_{6}, X_{1}, X_{2}\right)-\left\|X_{7}\right\|^{-2} \bar{\varphi}\left(T_{X_{7}} N, X_{7}, X_{1}, X_{2}\right) .
\end{aligned}
$$

Because of our choice of basis, the first and fourth terms on the right-hand side of this equation cancel, as do the second and third. Hence (4.8) follows.

We next specialize Theorems (4.8), (4.9) and (4.10) to the case where $\bar{M}=R^{7}$ or $R^{8}$ and $\bar{P}$ is the parallel translate of an ordinary (i.e., vector space instead of manifold) 2- or 3-fold vector cross product. Here the metric may be definite or indefinite. 
THEOREM (4.11). Let $M$ be an orientable hypersurface of $\bar{M}=R^{7}$ or $R^{8}$ with unit normal $N$ satisfying $\|N\|^{2}=1$, and let $P$ denote the vector cross product on $M$ determined by an ordinary vector cross product $\bar{P}$ on $\bar{M}$. Then

(i) $P$ is semiparallel;

(ii) if $P$ is almost parallel and $M$ is a 7-dimensional submanifold of $R^{8}$ with positive definite metric, then $M$ is an open submanifold of a hyperplane and $P$ is parallel;

(iii) if $P$ is nearly parallel, then up to an isometry of $R^{7}$ or $R^{8}, M$ is an open submanifold of the quadric $Q(r)=\left\{x \in M \mid\|x\|^{2}=r\right\}$ where $r>0$.

Proof. The parallel translate $\bar{P}$ of an ordinary vector cross product has covariant derivative zero. Hence (i) and (ii) follow easily from (4.9) and (4.10). Also, (iii) follows from Theorem (4.8) and the well-known fact that a hypersurface is totally umbilic if and only if it is an open submanifold of a quadric $Q(r)$.

In $\$ 7$ we prove theorems corresponding to Theorem (4.9) and Theorem (4.11) (ii) for almost complex structures.

It is now possible to determine the strictness of the inclusion relations between the classes $\mathscr{P}, \mathscr{N} \mathscr{P}, \mathscr{A} \mathscr{P}, \mathscr{S P}, \mathscr{Q} \mathscr{K}$, and $\mathscr{H}$. Let $<$ denote strict inclusion and let $\mathscr{M}(s, t, r)$ be the class of all manifolds possessing a vector cross product of type $(s, t, r)$; then $\mathscr{M}(s, t, 1)=\mathscr{A} \mathscr{H}(s, t)$.

THEOREM (4.13). (i) If $s$ and $t$ are integers such that $s+t=4$, then

$$
\begin{aligned}
& \mathscr{K}(s, t)<\mathscr{S} \mathscr{K}(s, t)<\mathscr{S} \mathscr{K}(s, t) \cup \mathscr{H}(s, t)<\mathscr{A} \mathscr{H}(s, t), \\
& \mathscr{K}(s, t)<\mathscr{H}(s, t)<\mathscr{S} \mathscr{K}(s, t) \cup \mathscr{H}(s, t)<\mathscr{A} \mathscr{H}(s, t) .
\end{aligned}
$$

(ii) If $s$ and $t$ are even integers such that $s+t \geqq 6$, then

$$
\begin{aligned}
\mathscr{K}(s, t) & <\mathscr{A} \mathscr{K}(s, t)<\mathscr{A} \mathscr{K}(s, t) \cup \mathscr{N} \mathscr{K}(s, t) \\
& <\mathscr{Q} \mathscr{K}(s, t) \cup(\mathscr{S} \mathscr{K}(s, t) \cap \mathscr{H}(s, t))<\mathscr{S} \mathscr{K}(s, t), \\
\mathscr{K}(s, t) & <\mathscr{N} \mathscr{K}(s, t)<\mathscr{A} \mathscr{K}(s, t) \cup \mathscr{N} \mathscr{K}(s, t) \\
& <\mathscr{Q} \mathscr{K}(s, t) \cup(\mathscr{S} \mathscr{K}(s, t) \cap \mathscr{H}(s, t))<\mathscr{S} \mathscr{K}(s, t)
\end{aligned}
$$

and

$$
\begin{aligned}
& \mathscr{K}(s, t)<\mathscr{S} \mathscr{K}(s, t) \cap \mathscr{H}(s, t)<\mathscr{S} \mathscr{K}(s, t)<\mathscr{S} \mathscr{K}(s, t) \cup \mathscr{H}(s, t)<\mathscr{A} \mathscr{H}(s, t), \\
& \mathscr{K}(s, t)<\mathscr{S} \mathscr{K}(s, t) \cap \mathscr{H}(s, t)<\mathscr{H}(s, t)<\mathscr{S} \mathscr{K}(s, t) \cup \mathscr{H}(s, t)<\mathscr{A} \mathscr{H}(s, t) .
\end{aligned}
$$

(iii) If $(s, t)=(0,7)$ or $(4,3)$, then

$\mathscr{P}(s, t, 2)<\mathscr{N} \mathscr{P}(s, t, 2)<\mathscr{S P}(s, t, 2)<\mathscr{M}(s, t, 2)$ and $\mathscr{A} \mathscr{P}(s, t, 2)<\mathscr{M}(s, t, 2)$.

(iv) If $(s, t)=(0,8),(4,4)$, or $(8,0)$, then

$$
\mathscr{S P}(s, t, 3)<\mathscr{M}(s, t, 3) \text { and } \mathscr{A} \mathscr{P}(s, t, 3)<\mathscr{M}(s, t, 3) .
$$

Proof. We omit the proofs of (i) and (ii) because they are essentially the same as a slightly weaker result of [11]. For (iii) the sphere $S^{7}$ and its indefinite analog $S_{4}^{3}$ show the strictness of the inclusion $\mathscr{P}(s, t, 2)<\mathscr{N} \mathscr{P}(s, t, 2)$. Furthermore if $M$ 
is a pseudo-Riemannian hypersurface of $R^{7}$ which is not an open submanifold of a hyperplane, sphere, or indefinite quadric, then $M$ is an example of a 7-dimensional manifold with a 2 -fold vector cross product which is semiparallel, but not nearly parallel. To prove the strict inclusions $\mathscr{S P}(s, t, 2)<\mathscr{M}(s, t, 2)$ and $\mathscr{A} \mathscr{P}(s, t, 2)$ $<\mathscr{M}(s, t, 2)$, let $M=R^{7}$ and give $M$ the usual metric with signature $(0,7)$ or $(4,3)$. Then if $M^{0}$ is conformally diffeomorphic but not homothetic to $R^{7}$, it follows from an argument similar to that of [11] that the induced vector cross product $P^{0}$ on $M^{0}$ is neither semiparallel nor almost parallel. The proof of (iv) is the same as that of (iii) with $R^{7}$ replaced by $R^{8}$.

5. Relations between curvature and vector cross products. In this section we generalize some theorems of Kotō [13]. As before let $M$ be a pseudo-Riemannian manifold with metric tensor $\langle$,$\rangle , and suppose P$ is an $r$-fold vector cross product on $M$ whose associated bilinear form is $\langle, \quad\rangle$. Also, we denote by $\varphi$ the differential form of degree $r+1$ determined by $\langle, \quad>$ and $P$.

Let $\theta$ be a $p$-form on $M$ and let $X, Y, X_{1}, \ldots, X_{p} \in \mathfrak{X}(M)$. In this section we shall need the following formulas:

$$
\begin{aligned}
& \nabla \theta\left(X ; X_{1}, \ldots, X_{p}\right)=\nabla_{X}(\theta)\left(X_{1}, \ldots, X_{p}\right) \\
& =X \theta\left(X_{1}, \ldots, X_{p}\right)-\sum_{i=1}^{p} \theta\left(X_{1}, \ldots, \nabla_{X} X_{i}, \ldots, X_{p}\right), \\
& \nabla^{2} \theta\left(X ; Y ; X_{1}, \ldots, X_{p}\right)=\nabla_{X}(\nabla \theta)\left(Y ; X_{1}, \ldots, X_{p}\right), \\
& R_{X Y}(\theta)\left(X_{1}, \ldots, X_{p}\right)=-\sum_{i=1}^{p} \theta\left(X_{1}, \ldots, R_{X Y} X_{i}, \ldots, X_{p}\right), \\
& \Delta \theta\left(X_{1}, \ldots, X_{p}\right)=\sum_{i=1}^{p} \sum_{k=1}^{n}(-1)^{i+1}\left\|E_{k}\right\|^{-2} R_{X_{i} E_{k}}(\theta)\left(E_{k}, X_{1}, \ldots, \hat{X}_{i}, \ldots, X_{p}\right) \\
& -\sum_{k=1}^{n}\left\|E_{k}\right\|^{-2} \nabla^{2} \theta\left(E_{k} ; E_{k} ; X_{1}, \ldots, X_{p}\right) \text {, }
\end{aligned}
$$

where $\left\{E_{1}, \ldots, E_{k}\right\}$ is an orthogonal frame field on an open subset of $M$. Here $\nabla \theta$ and $\nabla^{2} \theta$ are the first and second covariant derivatives of $\theta, R_{X Y}=\nabla_{[X, Y]}-\left[\nabla_{X}, \nabla_{Y}\right]$, and $\Delta=d \delta+\delta d$ is the Laplacian.

Proposition (5.1). Let $X, Y, Z_{1}, \ldots, Z_{r} \in \mathfrak{X}(M)$; then

$$
\begin{aligned}
\nabla^{2} \varphi\left(X ; Y ; Z_{1}, \ldots, Z_{r}, P\left(Z_{1}\right.\right. & \left.\left., \ldots, Z_{r}\right)\right) \\
& =-\left\langle\nabla_{X}(P)\left(Z_{1}, \ldots, Z_{r}\right), \nabla_{Y}(P)\left(Z_{1}, \ldots, Z_{r}\right)\right\rangle .
\end{aligned}
$$

Proof. This formula is an easy calculation from the definition of $\nabla^{2} \varphi$.

If $R_{X Y}$ denotes the curvature operator of $\langle, \quad\rangle$, then the Ricci curvature $k$ is defined by

$$
k(X, Y)=\sum_{i=1}^{n}\left\|E_{i}\right\|^{-2}\left\langle R_{X E_{i}} Y, E_{i}\right\rangle
$$


for $X, Y \in \mathfrak{X}(M)$, where $\left\{E_{1}, \ldots, E_{n}\right\}$ is any orthogonal frame field on an open subset of $M$. It will also be useful to consider a certain $(r+1)$-fold differential form $\gamma$.

Definition. The Chern form $\gamma$ of the vector cross product $P$ is defined by

$$
\begin{aligned}
& (r+1) \gamma\left(X_{1}, \ldots, X_{r+1}\right) \\
& \quad=\sum_{k=1}^{n} \sum_{i<j}(-1)^{r+i+j}\left\|E_{k}\right\|^{-2}\left\langle R_{X_{i} X_{j}} E_{k}, P\left(E_{k}, X_{1}, \ldots, \hat{X}_{i}, \ldots, \hat{X}_{j}, \ldots, X_{r+1}\right)\right\rangle
\end{aligned}
$$

for $X_{1}, \ldots, X_{r+1} \in \mathfrak{X}(M)$ and any orthogonal frame field $\left\{E_{1}, \ldots, E_{n}\right\}$.

It is not hard to verify that $\gamma$ is independent of the choice of $\left\{E_{1}, \ldots, E_{n}\right\}$; and is skew-symmetric, so that it is in fact a differential form. If $r=1$, so that $P$ is an almost complex structure $J$, then

$$
2 \gamma(X, Y)=\sum_{k=1}^{n}\left\|E_{k}\right\|^{-2}\left\langle R_{X Y} E_{k}, J E_{k}\right\rangle
$$

for $X, Y \in \mathfrak{X}(M)$. Hence if $J$ is Kählerian, then $\gamma(X, Y)=k(J X, Y)$ and $d \gamma=0$. Thus $\gamma$ determines the first Chern class in the usual sense for Kähler manifolds. (However, for general complex manifolds this is false.)

Proposition (5.2). Let $X_{1}, \ldots, X_{r+1} \in \mathfrak{X}(M)$, and let $\left\{E_{1}, \ldots, E_{n}\right\}$ be an orthogonal frame field on an open subset of $M$. Then

$$
\begin{aligned}
\Delta \varphi\left(X_{1}, \ldots, X_{r+1}\right)= & -\sum_{k=1}^{n}\left\|E_{k}\right\|^{-2} \nabla^{2} \varphi\left(E_{k} ; E_{k} ; X_{1}, \ldots, X_{r+1}\right) \\
& -\sum_{i=1}^{r+1}(-1)^{i+r} k\left(X_{i}, P\left(X_{1}, \ldots, X_{i}, \ldots, X_{r+1}\right)\right) \\
& -(r+1) \gamma\left(X_{1}, \ldots, X_{r+1}\right) .
\end{aligned}
$$

Proof. Formula (5.2) follows easily from the definitions of the Laplacian, the Ricci curvature, and the Chern form.

TheOREM (5.3). Let $X_{1}, \ldots, X_{r} \in \mathfrak{X}(M)$ and $\left\{E_{1}, \ldots, E_{n}\right\}$ be an orthogonal frame field on an open subset of $M$. Then

$$
\begin{aligned}
& \Delta \varphi\left(X_{1}, \ldots, X_{r}, P\left(X_{1}, \ldots, X_{r}\right)\right) \\
& =\sum_{k=1}^{n}\left\|E_{k}\right\|^{-2}\left\|\nabla_{E_{k}}(P)\left(X_{1}, \ldots, X_{r}\right)\right\|^{2} \\
& \quad-(r+1) \gamma\left(X_{1}, \ldots, X_{r}, P\left(X_{1}, \ldots, X_{r}\right)\right) \\
& \quad+k\left(P\left(X_{1}, \ldots, X_{r}\right), P\left(X_{1}, \ldots, X_{r}\right)\right) \\
& \quad+\sum_{i=1}^{r} \sum_{j=1}^{r}(-1)^{i+j}\left\langle X_{1} \wedge \cdots \wedge \hat{X}_{i} \wedge \cdots \wedge X_{r},\right. \\
& \left.\quad X_{1} \wedge \cdots \wedge \hat{X}_{j} \wedge \cdots \wedge X_{r}\right\rangle k\left(X_{i}, X_{j}\right) .
\end{aligned}
$$


Proof. The first sum on the right-hand side of (5.3) is the same as the first sum on the right-hand side of (5.2) by Proposition (5.1). Furthermore,

$$
-\sum_{i=1}^{r}(-1)^{i+r} k\left(X_{i}, P\left(X_{1}, \ldots, \hat{X}_{i}, \ldots, X_{r}\right)\right)
$$

is equal to the double sum on the right-hand side of (5.3) because $\left\|P\left(X_{1}, \ldots, X_{r}\right)\right\|^{2}$ $=\left\|X_{1} \wedge \cdots \wedge X_{r}\right\|^{2}$. Now Theorem (5.3) follows from Proposition (5.2).

If a vector cross product $P$ on a manifold $M$ is parallel then $\nabla_{X}(\varphi)=0$ for $X \in \mathfrak{X}(M)$ and so $R_{X Y}(\varphi)=0(X, Y \in \mathfrak{X}(M))$. This gives rise to certain identities that the curvature operator $R_{X Y}$ must satisfy. Conversely we have the following result for the class $\mathscr{A} \mathscr{P} \cap \mathscr{S} \mathscr{P}$.

Proposition (5.4). Suppose $P \in \mathscr{A} \mathscr{P} \cap \mathscr{S} \mathscr{P}$ and $\langle, \quad>$ is positive definite. If the curvature operator $R_{X Y}$ has all the symmetry properties that would be satisfied if $P$ were parallel, then $P$ is in fact parallel.

Proof. This result follows easily from the definition of $\Delta \varphi$, Proposition (5.1) and Proposition (5.3).

As as application of Proposition (5.4) we obtain a result of Tachibana [17]. The curvature operator of complex projective space (with the usual metric and almost complex structure) is given by the formula

$$
\begin{aligned}
\left\langle R_{W X} Y, Z\right\rangle= & K(\langle W, Y\rangle\langle X, Z\rangle-\langle W, Z\rangle\langle X, Y\rangle \\
& +\langle J W, Y\rangle\langle J X, Z\rangle-\langle J W, Z\rangle\langle J X, Y\rangle+2\langle J W, X\rangle\langle J Y, Z\rangle) .
\end{aligned}
$$

Let $M$ be a Riemannian almost complex manifold whose curvature operator is given by (5.4). Proposition (5.4) shows that if the almost complex structure $J$ of $M$ is almost Kählerian, then it is in fact Kählerian. The results at the end of this section show that a corresponding result holds for the class $\mathscr{N} \mathscr{K}$.

In a different direction we have the following two theorems for the case of positive definite metrics.

THEOREM (5.5). Suppose $P$ is a vector cross product on a Riemannian manifold $M$, $P$ is in $\mathscr{A} \mathscr{K}(0, n)(n \geqq 4), \mathscr{A} \mathscr{P}(0,7,2) \cap \mathscr{S} \mathscr{P}(0,7,2)$ or $\mathscr{A} \mathscr{P}(0,8,3) \cap \mathscr{S} \mathscr{P}(0,8,3)$, and $M$ is conformally flat. Denote by $k$ and $R$ the Ricci and Ricci scalar curvature of $M$.

(i) If $k$ is positive semidefinite on $M$, then it is identically zero and $P$ is parallel.

(ii) If $R \geqq 0$ on $M$, then $R$ vanishes identically on $M$ and $P$ is parallel.

Proof. Suppose $M$ is conformally flat. Then for $W, X, Y, Z \in \mathfrak{X}(M)$,

$$
\begin{aligned}
\left\langle R_{W X} Y, Z\right\rangle= & \frac{-R}{(n-1)(n-2)}(\langle W, Y\rangle\langle X, Z\rangle-\langle W, Z\rangle\langle X, Y\rangle) \\
& +\frac{1}{n-2}(k(W, Y)\langle X, Z\rangle-k(W, Z)\langle X, Y\rangle \\
& +k(X, Z)\langle W, Y\rangle-k(X, Y)\langle W, Z\rangle) .
\end{aligned}
$$


(Here $R=\sum_{i=1}^{n}\left\|E_{i}\right\|^{-2} k\left(E_{i}, E_{i}\right)$ for an orthogonal frame field $\left\{E_{1}, \ldots, E_{n}\right\}$.) From (5.5) it follows that if $X_{1}, \ldots, X_{r} \in \mathfrak{X}(M)$ are mutually orthogonal,

$$
\begin{gathered}
(r+1) \gamma\left(X_{1}, \ldots, X_{r}, P\left(X_{1}, \ldots, X_{r}\right)\right)=\frac{-r(r+1) R}{(n-1)(n-2)}\left\|X_{1} \wedge \cdots \wedge X_{r}\right\|^{2} \\
+\frac{2 r}{n-1} \sum_{i=1}^{r} k\left(X_{i}, X_{i}\right)\left\|X_{1} \wedge \cdots \wedge \hat{X}_{i} \wedge \cdots \wedge X_{r}\right\|^{2}
\end{gathered}
$$

Therefore, if we choose the $E_{k}$ 's in (5.3) so that $\left\|E_{k}\right\|^{2}=1$ we obtain

$$
\begin{aligned}
& \Delta \varphi\left(X_{1}, \ldots, X_{r}, P\left(X_{1}, \ldots, X_{r}\right)\right) \\
& =\sum_{k=1}^{n}\left\|\nabla_{E_{k}}(P)\left(X_{1}, \ldots, X_{r}\right)\right\|^{2} \\
& \quad+\frac{r(r+1) R}{(n-1)(n-2)}\left\|X_{1} \wedge \cdots \wedge X_{r}\right\|^{2}+k\left(P\left(X_{1}, \ldots, X_{r}\right), P\left(X_{1}, \ldots, X_{r}\right)\right) \\
& \quad+\left(1-\frac{2 r}{n-2}\right) \sum_{i=1}^{r} k\left(X_{i}, X_{i}\right)\left\|X_{1} \wedge \cdots \wedge X_{i} \wedge \cdots \wedge X_{r}\right\|^{2} .
\end{aligned}
$$

The hypotheses of the theorem imply that the left-hand side of (5.6) vanishes; hence (i) follows. Furthermore from (5.6) we obtain

$$
\begin{aligned}
\sum_{j_{1}}^{n} \sum_{, j_{r}=1}^{n} & \Delta \varphi\left(E_{j_{1}}, \ldots, E_{j_{r}}, P\left(E_{j_{1}}, \ldots, E_{j_{r}}\right)\right)=\sum_{k, j_{1}, \ldots, j_{r}=1}^{n}\left\|\nabla_{E_{k}}(P)\left(E_{j_{1}}, \ldots, E_{j_{r}}\right)\right\|^{2} \\
& +\frac{R n^{r-2}}{(n-1)(n-2)}\left\{(r+1) n^{3}-\left(r^{2}+3 r+2\right) n^{2}+\left(2 r^{2}+5 r-1\right) n-2(r-1)\right\} .
\end{aligned}
$$

Then (ii) follows from this equation.

THEOREM (5.6). Suppose $P$ is a vector cross product on a Riemannian manifold, and assume $P$ is in $\mathscr{A} \mathscr{K}(0, n)(n \geqq 4), \mathscr{A} \mathscr{P}(0,7,2) \cap \mathscr{S P}(0,7,2)$, or $\mathscr{A} \mathscr{P}(0,8,3)$ $\cap \mathscr{S} \mathscr{P}(0,8,3)$. If $M$ is a hypersurface of a flat Riemannian manifold $\bar{M}$, then $M$ cannot have positive Ricci scalar curvature.

Proof. Let $\left\{E_{1}, \ldots, E_{n}\right\}$ be an orthonormal frame field on an open subset of $M$ such that at a point $m$ each of the $E_{i}$ 's is an eigenvector of $X \rightarrow T_{X} N$ where $N$ is a unit normal to $M$ and $T$ is the configuration tensor of $M$. Write $T_{E_{i}} N=\kappa_{i} N$, $i=1, \ldots, n$. Then

$$
\left\langle R_{E_{i} E_{j}} E_{k}, E_{l}\right\rangle=\kappa_{i} \kappa_{j}\left(\delta_{i k} \delta_{j l}-\delta_{i l} \delta_{j k}\right),
$$

by the Gauss equation (see, e.g., [10]), and so

$$
(r+1) \gamma\left(E_{1}, \ldots, E_{r+1}\right)=\left(\sum_{i \neq j} \kappa_{i} \kappa_{j}\right) \varphi\left(E_{1}, \ldots, E_{r+1}\right)
$$


Thus (5.3) reduces to

$$
\begin{aligned}
\Delta \varphi\left(E_{1}, \ldots, E_{r}, P\left(E_{1}, \ldots, E_{r}\right)\right)= & \sum_{k=1}^{n}\left\|\nabla_{E_{k}}(P)\left(E_{1}, \ldots, E_{r}\right)\right\|^{2} \\
& +2 R+k\left(P\left(E_{1}, \ldots, E_{r}\right), P\left(E_{1}, \ldots, E_{r}\right)\right) \\
& +\sum_{i=1}^{r} k\left(E_{i}, E_{i}\right) .
\end{aligned}
$$

From this we obtain

$$
\begin{aligned}
\sum_{j_{1}, \ldots, j_{r}=1}^{n} & \Delta \varphi\left(E_{j_{1}}, \ldots, E_{j_{r}}, P\left(E_{j_{1}}, \ldots, E_{j_{r}}\right)\right) \\
& =n^{r-2}\left(2 n^{2}+(r+1) n-r+1\right) R+\sum_{k, j_{1}, \ldots, j_{r}=1}^{n}\left\|\nabla_{E_{k}}(P)\left(E_{j_{1}}, \ldots, E_{j_{r}}\right)\right\|^{2} .
\end{aligned}
$$

The theorem follows from this equation.

Next we look at the class $\mathscr{N} \mathscr{K}$.

THEOREM (5.7). Suppose $P$ is nearly parallel. Then for $X, Z_{1}, \ldots, Z_{r} \in \mathfrak{X}(M)$ we have

$$
\begin{aligned}
\left\|\nabla_{X}(P)\left(Z_{1}, \ldots, Z_{r}\right)\right\|^{2}= & \left\langle R_{X Z_{1}} X \wedge Z_{2} \wedge \cdots \wedge Z_{r}, Z_{1} \wedge \cdots \wedge Z_{r}\right\rangle \\
& +\sum_{i=2}^{r}\left\langle P\left(X, Z_{2}, \ldots, R_{X Z_{1}} Z_{i}, \ldots, Z_{r}\right), P\left(Z_{1}, \ldots, Z_{r}\right)\right\rangle \\
& -\left\langle R_{X Z_{1}} P\left(X, Z_{2}, \ldots, Z_{r}\right), P\left(Z_{1}, \ldots, Z_{r}\right)\right\rangle .
\end{aligned}
$$

Proof. It is not hard to see, using the fact that $P$ is nearly parallel, that

$$
\left\|\nabla_{X}(P)\left(Z_{1}, \ldots, Z_{r}\right)\right\|^{2}=-R_{X Z_{1}}(\varphi)\left(X, Z_{2}, \ldots, Z_{r}, P\left(Z_{1}, \ldots, Z_{r}\right)\right) .
$$

Expansion of the right-hand side of (5.9) yields the right-hand side of (5.8).

COROLLARY (5.8). The curvature operator of an almost Hermitian manifold whose almost complex structure is nearly parallel satisfies the identities

$$
\begin{aligned}
\left\langle\nabla_{W}(J)(X), \nabla_{Y}(J)(Z)\right\rangle & =\left\langle R_{W X} Y, Z\right\rangle-\left\langle R_{W X} J Y, J Z\right\rangle, \\
\left\langle R_{J W J X} J Y, J Z\right\rangle & =\left\langle R_{W X} Y, Z\right\rangle, \\
k(J W, J X) & =k(W, X), \\
\gamma(J W, J X) & =\gamma(W, X),
\end{aligned}
$$

for $W, X, Y, Z \in \mathfrak{X}(M)$.

Proof. As a special case of (5.8) we have

$$
\left\|\nabla_{X}(J)(Y)\right\|^{2}=\left\langle R_{X Y} X, Y\right\rangle-\left\langle R_{X Y} J X, J Y\right\rangle
$$


for $X, Y \in \mathfrak{X}(M)$. Linearization of this equation together with the first Bianchi identity yields

$$
\begin{aligned}
& 3\left\langle R_{W X} Y, Z\right\rangle-\left\langle R_{X Y} J X, J Z\right\rangle+\left\langle R_{W Z} J X, J Y\right\rangle-2\left\langle R_{W X} J Y, J Z\right\rangle \\
= & \left\langle\nabla_{W}(J)(Y), \nabla_{X}(J)(Z)\right\rangle-\left\langle\nabla_{W}(J)(Z), \nabla_{X}(J)(Y)\right\rangle+2\left\langle\nabla_{W}(J)(X), \nabla_{Y}(J)(Z)\right\rangle
\end{aligned}
$$

for $W, X, Y, Z \in \mathfrak{X}(M)$.

We replace $Y$ and $Z$ by $J Y$ and $J Z$ and subtract the result from (5.14). Since $\nabla_{U}(J)(V)+\nabla_{J U}(J)(J V)=0$ for all $U, V \in \mathfrak{X}(M)$, we obtain after some simplification

$$
\begin{aligned}
4\left\langle\nabla_{W}(J)(X),\right. & \left.\nabla_{Y}(J)(Z)\right\rangle \\
= & 5\left\langle R_{W X} Y, Z\right\rangle-5\left\langle R_{W X} J Y, J Z\right\rangle-\left\langle R_{W J X} J Y, Z\right\rangle-\left\langle R_{W X} Y, J Z\right\rangle .
\end{aligned}
$$

In (5.15) we replace $X$ and $Y$ by $J X$ and $J Y$ and add $1 / 5$ of the resulting equation to (5.15). We then obtain (5.10).

Two applications of (5.10) yield (5.11). Finally, (5.12) and (5.13) are easy consequences of (5.11).

Proposition (5.9). Suppose $P$ is an r-fold nearly parallel vector cross product, not of Type II, on a hypersurface $M$ of a conformally flat pseudo-Riemannian manifold $\bar{M}$. Assume that the normal bundle of $M$ in $\bar{M}$ has positive definite metric, assume all the eigenvalues of $X \rightarrow T_{X} N$ are real and determine $X \rightarrow T_{X} N$, and let the signature of $M$ be $(s, t)$. Let $K_{+}$and $K_{-}$be the restrictions of the sectional curvature of $M$ to fields of 2-planes on which the metric of $M$ is positive definite and negative definite respectively. Then

(i) $s \leqq r+1$ implies $(-1)^{s} K_{-} \geqq 0$;

(ii) $t \leqq r+1$ implies $(-1)^{t+r} K_{+} \geqq 0$;

(iii) $s \leqq r-1$ implies $(-1)^{s} K_{+} \geqq 0$;

(iv) $t \leqq r-1$ implies $(-1)^{t+r} K_{-} \geqq 0$;

(v) $K_{+}=K_{-}=0$ if $P$ is parallel.

Proof. Let $\bar{R}_{X Y}, \bar{k}$, and $\bar{R}$ denote the curvature operator, Ricci curvature, and Ricci scalar curvature of $\bar{M}$, and denote by $T$ the configuration tensor field of $M$ in $\bar{M}$. Choose a unit normal $N$ on an open subset of $M$ and let $W, X, Y, Z$ lie in eigenspaces of $X \rightarrow T_{X} N$ at a point $m \in M$, and denote the corresponding eigenvalues by $\kappa_{W}, \kappa_{X}, \kappa_{Y}$ and $\kappa_{Z}$. From (5.5) and (5.7) it follows that the curvature operator $R_{X Y}$ of $M$ is given by the formula

$$
\left\langle R_{W X} Y, Z\right\rangle=\left(\kappa_{W} \kappa_{X}-\frac{\bar{R}}{n(n-1)}\right)(\langle W, Y\rangle\langle X, Z\rangle-\langle W, Z\rangle\langle X, Y\rangle)
$$

$$
\begin{aligned}
+\frac{1}{n-1}(\bar{k}(W, Y)\langle X, Z\rangle-\bar{k}(W, Z)\langle X, Y\rangle & \\
+ & +\bar{k}(X, Z)\langle W, Y\rangle-\bar{k}(X, Y)\langle W, Z\rangle),
\end{aligned}
$$


where $\operatorname{dim} M=n$. In particular $\left\langle R_{W X} Y, Z\right\rangle=0$ if $W, X, Y, Z$ are mutually orthogonal. Choose $X, Z_{1}, \ldots, Z_{r} \in \mathfrak{X}(M)$ to be linearly independent eigenvectors of $X \rightarrow T_{X} N$ at $m \in M$. Then from (5.8) and (5.16) we have

$$
\left\|\nabla_{X}(P)\left(Z_{1}, \ldots, Z_{r}\right)\right\|^{2}=K_{X Z_{1}}\|X\|^{2}\left\|Z_{1}\right\|^{2} \cdots\left\|Z_{r}\right\|^{2}
$$

where $K_{X Z_{1}}$ denotes the sectional curvature of the plane spanned by $X$ and $Z_{1}$. Here we require that $\left\langle P\left(Z_{1}, \ldots, Z_{r}\right), X\right\rangle=0$.

If $s \leqq r+1$, choose $X, Z_{1}, \ldots, Z_{s-1}$ to have negative norms. Then

$$
\nabla_{X}(P)\left(Z_{1}, \ldots, Z_{r}\right)
$$

has a nonnegative norm, since it is orthogonal to $X, Z_{1}, \ldots, Z_{s-1}$. Now (5.17) implies (i). Furthermore the same type of argument yields (ii)-(v).

Next we specialize Proposition (5.9) to vector cross products of each of Types I, III, and IV.

THEOREM (5.10). Let $J$ be a nearly Kähler almost complex structure on an ndimensional manifold $M$ which satisfies the hypotheses of Proposition (5.9). Then

(i) $K_{-} \geqq 0$ if $M$ has signature $(2, n-2)$;

(ii) $K_{+} \leqq 0$ if $M$ has signature $(n-2,2)$;

(iii) $K \geqq 0$ if $M$ has signature $(0, n)$;

(iv) $K \leqq 0$ if $M$ has signature $(n, 0)$;

(v) $K_{+}=K_{-}=0$ if $J$ is Kählerian;

(vi) $J$ is Kählerian if $K_{+}=K_{-}=0$ and $M$ has signature $(0, n),(n, 0),(2,2),(2,4)$ or $(4,2)$.

Proof. (i)-(v) are immediate from Proposition (5.9) and (vi) is obvious if $M$ has signature $(0, n),(n, 0)$, or $(2,2)$. Suppose $M$ has signature $(2,4)$ or $(4,2)$. Choose an orthogonal basis on an open subset of $M$ of the form $\left\{X_{1}, J X_{1}, X_{2}, J X_{2}, X_{3}, J X_{3}\right\}$. Then if $i, j, k$ are not equal, $\nabla_{X_{i}}(J)\left(X_{j}\right)$ is a linear combination of $X_{k}$ and $J X_{k}$. By (5.17) we have $\nabla_{X_{i}}(J)\left(X_{j}\right)=0$. Thus $J$ is Kählerian.

THEOREM (5.11). Let $P$ be a nearly parallel 2-fold vector cross product on a 7dimensional manifold $M$ which satisfies the hypotheses of Proposition (5.9). Then

(i) $M$ has constant sectional curvature $K$;

(ii) $K \geqq 0$ if $M$ has signature $(0,7)$;

(iii) $K \leqq 0$ if $M$ has signature $(4,3)$;

(iv) $P$ is parallel if and only if $K=0$.

Proof. (i) follows from (5.17), and (ii) and (iii) are special cases of Proposition (5.9). The sufficiency of (iv) is clear; to prove the necessity let $\left\{X_{1}, \ldots, X_{7}\right\}$ be an orthogonal basis on an open subset of $M$ such that $X_{3}=P\left(X_{1}, X_{2}\right), X_{5}=P\left(X_{1}, X_{4}\right)$, $X_{6}=P\left(X_{2}, X_{4}\right)$, and $X_{7}=P\left(X_{3}, X_{4}\right)$. From Proposition (4.3) and the hypothesis that $P$ is nearly parallel it follows that $\nabla_{X_{i}}(P)\left(X_{j}, X_{k}\right)=\rho X_{l}$ for all $i, j, k$ for some $l$ and $\rho$. By (5.17) we have $\left\|\nabla_{X_{i}}(P)\left(X_{j}, X_{k}\right)\right\|^{2}=0$ and so $\nabla_{X_{i}}(P)\left(X_{j}, X_{k}\right)=0$. Thus $P$ is parallel. 
THEOREM (5.12). Let $P$ be a nearly parallel 3-fold vector cross product on an 8-dimensional manifold $M$ which satisfies the hypotheses of Proposition (5.9). Then $M$ is flat.

Proof. By Theorem (4.6), $P$ is parallel. Hence by Proposition (5.9) (iii), $M$ is flat.

6. Six-dimensional almost complex manifolds defined by means of a 3-fold vector cross product. This section and the rest of the paper are devoted to the most important kind of vector cross products on manifolds, namely almost complex structures. We shall use Theorem (2.6) to generate an interesting class of 6-dimensional almost complex manifolds. Then we study these manifolds by means of the configuration tensor $T$ (i.e., the second fundamental form).

Let $\bar{M}$ be a nondegenerate pseudo-Riemannian manifold of dimension 8 which possesses a 3-fold vector cross product $P$. Then the metric tensor $\langle, \quad>$ of $\bar{M}$ is positive definite, negative definite, or has the signature $(4,4)$. The case of a negative definite metric is essentially the same as that of a positive definite metric, and so we shall henceforth exclude it from our discussion. Now suppose $M$ is a nondegenerate 6-dimensional submanifold of $\bar{M}$ such that the restriction of the metric tensor of $\bar{M}$ to the normal bundle of $M$ is positive definite. Then the metric tensor of $M$ is positive definite or has signature $(4,2)$.

Assume that $M$ is orientable. Then the normal bundle of $M$ in $\bar{M}$ is orientable since $\bar{M}$ is orientable. Since the normal bundle is 2-dimensional it has an almost complex structure $J$ defined on it. If we choose a unit normal $N$ on an open subset of $M$, then $N \wedge J N$ is independent of $N$ and may be extended to a global vector 2-field on $M$; then $\|N \wedge J \dot{N}\|^{2}=1$.

Definition. Let $P$ denote the vector cross product of $\bar{M}$. Then $J: \mathfrak{X}(M) \rightarrow \mathfrak{X}(M)$ is defined by $J A=P(N, J N, A)$ for $A \in \mathfrak{X}(M)$.

That $J$ is actually an almost complex structure on $M$ is a consequence of Theorem (2.6). Note that we have actually defined an almost complex structure on the Whitney sum of the tangent bundle and the normal bundle of $M$. Before proceeding further it will be necessary to derive some formulas involving $P$ and $J$.

Proposition (6.1). Let $A, B \in \mathfrak{X}(M)$ and let $N$ be a unit normal on an open subset of $M$. Then

$$
\begin{aligned}
& P(J A, B, N)+J P(A, B, N)=-\langle J A, B\rangle N-\langle A, B\rangle J N ; \\
& P(J A, J B, N)+P(A, B, N)=2\langle J A, B\rangle N .
\end{aligned}
$$

Furthermore, exactly one of the following equations holds (depending on the kind of 3-fold vector cross product on $\bar{M}$ ) for a given vector cross product $P$.

$$
\begin{aligned}
& J P(N, A, B)=P(J N, A, B) ; \\
& J P(N, A, B)=-P(J N, A, B)-2\langle J A, B\rangle N .
\end{aligned}
$$


Proof. To prove (6.1) we first observe that

$$
\begin{aligned}
\langle P(A, J A, N), B\rangle & =\langle P(N, B, A), P(N, J N, A)\rangle \\
& =\frac{1}{2}\left\{\|P(N, B+J N, A)\|^{2}-\|P(N, J N, A)\|^{2}-\|P(N, B, A)\|^{2}\right\} \\
& =\frac{1}{2}\left\{\|A\|^{2}\left(\|B\|^{2}+1\right)-\langle A, B\rangle^{2}-\|A\|^{2}-\|A\|^{2}\|B\|^{2}+\langle A, B\rangle^{2}\right\} \\
& =0 .
\end{aligned}
$$

Hence for $C \in \mathfrak{X}(M)$ we have

$$
\begin{aligned}
\langle P(J A, B, N) & +J P(A, B, N), C\rangle=\langle P(C, J A, N)+P(A, J C, N), B\rangle \\
& =\frac{1}{2}\langle P(A+C, J(A+C), N)-P(A, J A, N)-P(C, J C, N), B\rangle=0 .
\end{aligned}
$$

Furthermore

$$
\langle P(J A, B, N)+J P(A, B, N), N\rangle=-\langle J A, B\rangle
$$

and

$$
\langle P(J A, B, N)+J P(A, B, N), J N\rangle=-\langle A, B\rangle .
$$

Hence (6.1) follows; (6.2) is an easy consequence of (6.1).

To prove that one of (6.3) and (6.4) holds we may assume (on account of (6.1)) that $A, J A, B, J B$, and $P(J N, A, B)$ are linearly independent. Then both $J P(J N, A, B)$ and $P(N, A, B)$ are orthogonal to these five vector fields. Furthermore

$$
\langle J P(J N, A, B), J N\rangle=-\langle J A, B\rangle=-\langle P(N, A, B), J N\rangle .
$$

Hence there exist functions $\alpha$ and $\beta$ on $M$ such that

$$
\alpha\{J P(J N, A, B)+\langle J A, B\rangle J N\}=\beta\{P(N, A, B)-\langle J A, B\rangle J N\} .
$$

Taking norms of both sides of equation (6.5) we find that $\alpha= \pm \beta$. If $\alpha=-\beta$ then (6.3) holds, and if $\alpha=+\beta$ then (6.4) holds.

Now suppose both (6.3) and (6.4) hold. Then $P(N, A, B)=\langle J A, B\rangle J N$. It follows upon taking norms of both sides of this equation that $\|A\|^{2}\|B\|^{2}-\langle A, B\rangle^{2}=$ $\langle J A, B\rangle^{2}$. However, this equation is false whenever $A, B, J A, J B$ are nonisotropic and orthogonal.

For manifolds with a Cayley multiplication we can say more about the almost complex structures induced from 3 -fold vector cross products.

Proposition (6.2). Let $\bar{M}$ be an 8-dimensional pseudo-Riemannian manifold with a differentiable Cayley multiplication, and let $M$ be a nondegenerate orientable 6-dimensional submanifold of $\bar{M}$ such that the restriction of the metric tensor of $\bar{M}$ to the normal bundle of $M$ is positive definite. Let $P_{1}$ and $P_{2}$ be the 3-fold vector cross products on $\bar{M}$ which are defined by Cayley multiplication and are explicitly given by (2.3) and (2.4) with $\varepsilon=1$, respectively.

(i) $P_{1}$ defines an almost complex structure on $M$ which satisfies (6.3).

(ii) $P_{2}$ defines an almost complex structure on $M$ which satisfies (6.4). 
(iii) The almost complex structures on $M$ defined by $P_{1}$ and $P_{2}$ coincide if and only if $M$ is always normal to the identity vector field of the Cayley multiplication.

Proof. We prove (ii); the proof of (i) is similar. We have

$$
P_{2}(X, Y, Z)=-(X \cdot \sigma Y) \cdot Z+\langle X, Y\rangle Z+\langle Y, Z\rangle X-\langle X, Z\rangle Y
$$

for all $X, Y, Z \in \mathfrak{X}(\bar{M})$. Here $\sigma$ denotes the conjugation defined by means of the Cayley multiplication. Let $A, B \in \mathfrak{X}(M)$ and let $N$ be a unit normal on an open subset of $M$. Then

$$
\begin{aligned}
J P(N, A, B)+\langle J A, B\rangle N & =P_{2}\left(P_{2}(A, B, N), N, J N\right) \\
& =-\left(P_{2}(A, B, N) \cdot \sigma N\right) \cdot J N-\left\langle P_{2}(A, B, N), J N\right\rangle N \\
& =(((A \cdot \sigma B) \cdot N-\langle A, B\rangle N) \cdot \sigma N) \cdot J N-\langle J A, B\rangle N \\
& =(A \cdot \sigma B) \cdot J N-\langle A, B\rangle J N-\langle J A, B\rangle N \\
& =-P_{2}(A, B, J N)-\langle J A, B\rangle N .
\end{aligned}
$$

Hence (6.4) holds.

To prove (iii) we first note that the almost complex structures $J_{1}$ and $J_{2}$ defined by $P_{1}$ and $P_{2}$ respectively are given by the formulas

$$
J_{1} A=-N \cdot(\sigma J N \cdot A) ; \quad J_{2} A=-(N \cdot \sigma J N) \cdot A,
$$

for $A \in \mathfrak{X}(M)$. Hence $J_{1}=J_{2}$ if and only if

$$
(N \cdot \sigma J N) \cdot A=N \cdot(\sigma J N \cdot A)
$$

for all $A \in \mathfrak{X}(M)$. From (6.6) it follows that the identity vector field $E$ cannot be orthogonal to both $N$ and $J N$. Hence

$$
E=a N+b J N+c C
$$

where $a, b, c$ are functions on $M$ and $C \in \mathfrak{X}(M)$, and, say, $b \neq 0$. Substitution of (6.7) into (6.6) shows that $c=0$, and so $M$ is normal to $E$.

Conversely if $M$ is always normal to $E$ we may take $N=E$ on $M$. Then (6.6) holds and so $J_{1}=J_{2}$.

Let $M$ be an orientable 6-dimensional submanifold of $R^{8}$. Furthermore, let the Cayley multiplication at the origin of $R^{8}$ be translated over all of $R^{8}$ in the natural way. It is easy to see that if $M$ is contained in the hyperplane of pure Cayley numbers (i.e., those perpendicular to the identity) then the almost complex structures on $M$ defined by means of the two 3 -fold vector cross products are the same and coincide with Calabi's almost complex structure [6]. On the other hand if $M$ is not contained in the hyperplane of pure Cayley numbers, then by Proposition (6.2) the almost complex structures defined by the two 3-fold vector cross products are different. In particular, on $S^{6}$ we obtain some new almost complex structures which are almost Hermitian with respect to the natural metric of $S^{6}$. 
We next consider the problem of giving conditions, in terms of the configuration tensor $T$, that the almost complex structure $J$ defined by $J A=P(N, J N, A)$ be in one of the classes $\mathscr{K}, \mathscr{N} \mathscr{K}, \mathscr{A} \mathscr{K}, \mathscr{Q} \mathscr{K}, \mathscr{S} \mathscr{K}$, or $\mathscr{H}$. The following lemma will be useful.

LEMMA (6.3). Let $L: \mathfrak{X}(M)^{\perp} \rightarrow \mathfrak{X}(M)$ be a pointwise linear map. Then for $A, B \in \mathfrak{X}(M)$ and $Z \in \mathfrak{X}(M)^{\perp}$ we have

$$
\langle P(L Z, J Z, A)+P(Z, L J Z, A), B\rangle=\langle P(Z, L J Z \pm J L Z, A), B\rangle .
$$

Proof. We have by (6.3), (6.4), and (6.1),

$$
\begin{aligned}
\langle P(L Z, J Z, A)+P(Z, L J Z, A), B\rangle & =\langle\mp J P(Z, L Z, A)+P(Z, L J Z, A), B\rangle \\
& =\langle P(Z, L J Z \pm J L Z, A), B\rangle .
\end{aligned}
$$

In this lemma we have used " \pm " and " $\mp$ " in the following senses. If (6.3) holds " \pm " is to be interpreted " + " and " $\mp$ " is to be interpreted as " - ". If (6.4) holds, then " \pm " and " $\mp "$ are to be interpreted in the opposite way. We shall continue to use these conventions.

The classes $\mathscr{K}, \mathscr{H}$, and $\mathscr{S} \mathscr{K}$ are the easiest to deal with, and so we consider them first. As before we denote by $F$ and $\varphi$ the differential forms on $M$ and $\bar{M}$ associated with $J$ and $P$.

THEOREM (6.4). Suppose $P \in \mathscr{P}$. Then $J \in \mathscr{K}$ if and only if

$$
T_{A} J B \pm J T_{A} B=0
$$

for all $A, B \in \mathfrak{X}(M)$.

Proof. Let $A, B, C \in \mathfrak{X}(M)$ and let $N$ be a unit normal on an open subset of $M$. Then

$$
\begin{aligned}
\nabla_{A}(F)(B, C) & =\left\langle\bar{\nabla}_{A} P(N, J N, B)-P\left(N, J N, \bar{\nabla}_{A} B\right), C\right\rangle \\
& =\left\langle P\left(T_{A} N, J N, B\right)+P\left(N, T_{A} J N, B\right), C\right\rangle+\bar{\nabla}_{A}(\varphi)(N, J N, B, C) .
\end{aligned}
$$

Let $L Z=T_{A} Z$ for $Z \in \mathfrak{X}(M)^{\perp}$. Then by Lemma (6.3) we have

$$
\nabla_{A}(F)(B, C)=\bar{\nabla}_{A}(\varphi)(N, J N, B, C)+\left\langle P\left(N, T_{A} J N \pm J T_{A} N, B\right), C\right\rangle .
$$

If $P \in \mathscr{P}$ then the first term on the right-hand side of (6.10) vanishes. Now $J \in \mathscr{K}$ if and only if $\nabla_{A}(F)(B, C)=0$ for all $A, B, C \in \mathfrak{X}(M)$. By (6.10) this is equivalent to the condition that $T_{A} J N \pm J T_{A} N=0$ for all $A \in \mathfrak{X}(M)$ and all unit normals $N$. This last condition is equivalent to (6.9).

THEOREM (6.5). Suppose $P \in \mathscr{P}$. Then $J \in \mathscr{H}$ if and only if

$$
T_{A} A+T_{J A} J A=0
$$

for $A \in \mathfrak{X}(M)$. 
Proof. If $P \in \mathscr{P}$, then it follows from (6.10) and (6.2) that for $A, B, C \in \mathfrak{X}(M)$,

$$
\begin{aligned}
\nabla_{A}(F)(B, C)-\nabla_{J A}(F) & (J B, C) \\
& =\left\langle P\left(N, T_{A} J N-J T_{J A} J N \pm\left(J T_{A} N+T_{J A} N\right), B\right), C\right\rangle .
\end{aligned}
$$

The condition $J \in \mathscr{H}$ is equivalent [11] to the vanishing of the left-hand side of (6.12). Hence $J \in \mathscr{H}$ if and only if

$$
J\left(T_{A} B+T_{J A} J B\right) \pm\left(T_{A} J B-T_{J A} B\right)=0
$$

for all $A, B \in \mathfrak{X}(M)$. Then (6.13) implies (6.11). On the other hand, linearization of (6.11) yields $T_{A} B+T_{J A} J B=0$; this implies (6.13).

The mean curvature vector $H$ of $M$ in $\bar{M}$ is defined by $H=\sum_{i=1}^{6}\left\|E_{i}\right\|^{-2} T_{E_{i}} E_{i}$, where $\left\{E_{1}, \ldots, E_{6}\right\}$ is any nondegenerate orthogonal basis on an open subset of $M$. We say that $M$ is a minimal variety of $\bar{M}$ if $H$ vanishes identically on $M$. The holomorphic curvature of an almost complex manifold $M$ is the restriction of the sectional curvature of $M$ to fields of 2-planes spanned by vector fields $A$ and $J A$.

Corollary (6.6). Suppose $P \in \mathscr{P}$ and $J \in \mathscr{H}$. Then

(i) $M$ is a minimal variety of $\bar{M}$;

(ii) if $R_{A B}$ and $\bar{R}_{A B}$ denote the curvature operators of $M$ and $\bar{M}$ respectively, then for $A, B, C, D \in \mathfrak{X}(M)$

$$
\left\langle R_{A B} C, D\right\rangle-\left\langle R_{J A J B} J C, J D\right\rangle=\left\langle\bar{R}_{A B} C, D\right\rangle-\left\langle\bar{R}_{J A J B} J C, J D\right\rangle ;
$$

(iii) if $\bar{M}$ is flat, then the Ricci curvature $k$ and Chern form $\gamma$ of $M$ satisfy the relations

$$
k(J A, J B)=k(A, B)=\gamma(J A, B)=-\gamma(A, J B)
$$

for $A, B \in \mathfrak{X}(M)$;

(iv) on holomorphic fields of 2-planes tangent to $M$ the holomorphic curvature is less than or equal to the corresponding sectional curvature of $\bar{M}$.

Proof. (i) follows from (5.11), (ii) is an application of the Gauss equation [10], and (iii) follows from (ii). For (iv) we have by the Gauss equation that for $A \in \mathfrak{X}(M)$

$$
K_{A J A}=\left\langle T_{A} A, T_{J A} J A\right\rangle-\left\|T_{A} J A\right\|^{2}+\bar{K}_{A J A}=-\left\|T_{A} A\right\|^{2}-\left\|T_{A} J A\right\|^{2}+\bar{K}_{A J A} .
$$

The formulas of Corollary (6.6) (iii) do not hold for arbitrary Hermitian manifolds, e.g., the product of two odd-dimensional spheres of different dimension. We note that if $J \in \mathscr{K}$, the first two terms on the right-hand side of (6.15) are the same.

Corollary (6.7). Suppose $P \in \mathscr{P}, J \in \mathscr{H}$, and the metric of $\bar{M}$ is positive definite. If the sectional curvature of $\bar{M}$ is nonpositive, then $M$ is noncompact.

Proof. By Corollary (6.6) $M$ is a minimal variety. This implies (e.g., O'Neill [15]) that $M$ cannot be compact.

THEOREM (6.8). If $P \in \mathscr{S} \mathscr{P}$, then $J \in \mathscr{S} \mathscr{K}$. In particular if $\bar{M}=R^{8}$ and $P$ is the ordinary vector cross product on $\bar{M}$, then we always have $J \in \mathscr{S} \mathscr{K}$. 
Proof. It is possible to give a direct proof; however, the following is simpler. It suffices to prove the theorem locally. Therefore, locally at least, we can find an orientable 7-dimensional manifold $M^{\prime}$ such that $M \subset M^{\prime} \subset \bar{M}$. By Theorem (2.6), $M^{\prime}$ has a 2-fold vector cross product $P^{\prime}$ and by (4.10), $P^{\prime} \in \mathscr{S} \mathscr{P}$. Furthermore the almost complex structure that $P^{\prime}$ induces on $M$ is $J$. Again by (4.10), $J \in \mathscr{S} \mathscr{K}$.

Next we consider the class $\mathscr{2} \mathscr{K}$.

TheOrem (6.9). Suppose $P \in \mathscr{P}$. Then $J \in \mathscr{Q} \mathscr{K}$ if and only if for all $A \in \mathfrak{X}(M)$

$$
J\left(T_{A} A-T_{J A} J A\right) \pm 2 T_{A} J A=0 .
$$

Proof. We have

$$
\nabla_{A}(F)(B, C)+\nabla_{J A}(F)(J B, C)=\left\langle P\left(N, T_{A} J N+J T_{J A} J N \pm\left(J T_{A} N-T_{J A} N\right), B\right), C\right\rangle .
$$

The rest of the proof is, except for a few sign changes, the same as the proof of Theorem (6.5), and so we omit it.

Proposition (6.10). Suppose $P \in \mathscr{P}$ and $J \in \mathscr{Q} \mathscr{K}$. Then the holomorphic curvature of $M$ is given by the formula

$$
2 K_{A J A}=\left\|T_{A} A\right\|^{2}+\left\|T_{J A} J A\right\|^{2}-6\left\|T_{A} J A\right\|^{2}+2 \bar{K}_{A J A}
$$

for $A \in \mathfrak{X}(M)$, with $\|A\|^{2}= \pm 1$.

Proof. This follows from the Gauss equation and Theorem (6.9).

Next we derive a formula for quasi-Kähler manifolds involving the covariant derivative $\bar{\nabla}_{A}(T)$ of the configuration tensor $T$. This will be useful in $\S 7$. Here (see [12])

$$
\bar{\nabla}_{A}(T)_{B} W=\bar{\nabla}_{A}\left(T_{B} W\right)-T_{\nabla_{A} B} W-T_{B} \bar{\nabla}_{A} W
$$

for $A, B \in \mathfrak{X}(M)$ and $W \in \overline{\mathfrak{X}}(M)$. Also, for $X, Y, Z \in \overline{\mathfrak{X}}(M)$ we denote by $\bar{R}_{X Y} Z$ the curvature operator of $\bar{M}$.

TheOREM (6.11). Suppose $P \in \mathscr{P}$ and $J \in \mathscr{Q} \mathscr{K}$. Then for $A, B \in \mathfrak{X}(M)$ and all unit normals $N$ we have

$$
\begin{aligned}
& \left\langle\bar{\nabla}_{B}(T)_{A} A+\bar{\nabla}_{B}(T)_{J A} J A, N\right\rangle \pm\left\langle\bar{\nabla}_{J B}(T)_{A} A+\bar{\nabla}_{J B}(T)_{J A} J A, J N\right\rangle \\
& \quad=\left\langle\bar{R}_{A B} A+\bar{R}_{J A B} J A-\bar{R}_{A J A} J B, N\right\rangle \pm\left\langle\bar{R}_{A J B} A+\bar{R}_{J A J B} J A+\bar{R}_{A J A} B, J N\right\rangle .
\end{aligned}
$$

Proof. Linearization of (6.16) yields

$$
\left\langle T_{B} C-T_{J B} J C, N\right\rangle \pm\left\langle T_{B} J C+T_{J B} C, J N\right\rangle=0
$$

for $B, C \in \mathfrak{X}(M)$. If we apply $A \in \mathfrak{X}(M)$ to the left-hand side of (6.20) it follows, since $P \in \mathscr{P}$, that

$$
\begin{aligned}
\left\langle\bar{\nabla}_{A}(T)_{B} C-\bar{\nabla}_{A}(T)_{J B} J C, N\right\rangle \pm\left\langle\bar{\nabla}_{A}(T)_{B} J C+\bar{\nabla}_{A}(T)_{J B} C, J N\right\rangle \\
=\left\langle P\left(N, T_{A} J N \pm J T_{A} N, B\right),-T_{J C} N \pm T_{C} J N\right\rangle \\
\quad+\left\langle P\left(N, T_{A} J N \pm J T_{A} N, C\right),-T_{J B} N \pm T_{B} J N\right\rangle
\end{aligned}
$$


Letting $A=C$ in (6.21) and using the Codazzi equation [12]

$$
\left\langle\bar{\nabla}_{A}(T)_{B} C-\bar{\nabla}_{B}(T)_{A} C, N\right\rangle=-\left\langle\bar{R}_{A B} C, N\right\rangle,
$$

we obtain

$$
\begin{aligned}
\left\langle\bar{\nabla}_{B}(T)_{A} A-\bar{\nabla}_{J B}(T)_{A} J A, N\right\rangle \pm\left\langle\bar{\nabla}_{B}(T)_{A} J A+\bar{\nabla}_{J B}(T)_{A} A, J N\right\rangle \\
=\left\langle\bar{R}_{A B} A-\bar{R}_{A J B} J A, N\right\rangle \pm\left\langle\bar{R}_{A B} J A-\bar{R}_{A J B} A, J N\right\rangle \\
-\left\langle P\left(N, T_{A} J N \pm J T_{A} N, B\right), T_{J A} N \mp T_{A} J N\right\rangle \\
-\left\langle P\left(N, T_{A} J N \pm J T_{A} N, A\right), T_{J B} N \mp T_{B} J N\right\rangle
\end{aligned}
$$

Next we replace $A$ by $J A$ in (6.22) and add the result to (6.22); this yields (6.19).

Corollary (6.12). Suppose $P \in \mathscr{P}, J \in \mathscr{Q} \mathscr{K}$, and $\bar{M}$ is flat. Then the mean curvature vector $H$ of $M$ satisfies the equation

$$
B\|H\|^{2}=\mp 2\left\langle\bar{\nabla}_{J B}(H), J H\right\rangle
$$

for all $B \in \mathfrak{X}(M)$.

Proof. If $\bar{M}$ is flat then the right-hand side of (6.19) vanishes. Hence from (6.19) we obtain

$$
\left\langle\nabla_{B}(H), N\right\rangle \pm\left\langle\bar{\nabla}_{J B}(H), J N\right\rangle=0 .
$$

We may replace $N$ by $H$ in (6.24); this yields (6.23).

We now turn to the class $\mathscr{N} \mathscr{K}$.

Theorem (6.13). Suppose $P \in \mathscr{P}$. Then $J \in \mathscr{N} \mathscr{K}$ if and only if there exists a 1 -form $\beta$ on the normal bundle of $M$ such that

$$
T_{A} J N \pm J T_{A} N=\beta(J N) A \pm \beta(N) J A
$$

for all $A \in \mathfrak{X}(M)$.

Proof. We have

$$
\nabla_{A}(F)(A, B)=\left\langle P\left(N, T_{A} N \pm J T_{A} N, A\right), B\right\rangle
$$

for $A, B \in \mathfrak{X}(M)$. Assume that $J \in \mathscr{N} \mathscr{K}$. Then

$$
T_{A} J N \pm J T_{A} N=\beta(A, J N) A+\alpha(A, J N) J A .
$$

Since $J \in \mathscr{Q} \mathscr{K}$, it follows from (6.17) and (6.27) that $\alpha(A, J N)= \pm \beta(A, N)$ and that $\beta$ does not depend on $A$. It is then obvious from (6.27) that $\beta$ is linear on the normal bundle.

Conversely, if (6.25) holds, then it follows from (6.26) that $J \in \mathscr{N} \mathscr{K}$.

COROLlaRY (6.14). Suppose that equation (6.25) holds. Then the mean curvature vector field $H$ is dual to $-6 \beta$. 
Proof. From (6.25) it follows that $\|A\|^{-2}\left\langle T_{A} A+T_{J A} J A, N\right\rangle=-2 \beta(N)$. From this the corollary is immediate.

Using Theorem (6.10) we obtain in the next theorem a formula involving the covariant derivative $\bar{\nabla}_{B}(\beta)(B \in \mathfrak{X}(M))$. Here $\bar{\nabla}_{B}(\beta)(N)=B \beta(N)-\beta\left(\bar{\nabla}_{B} N\right)$.

TheOREM (6.15). Suppose $P \in \mathscr{P}$ and $J \in \mathscr{N} \mathscr{K}$. Then for $A, B \in \mathfrak{X}(M)$ and all unit normals $N$ we have

$$
\begin{aligned}
& 2\left(\bar{\nabla}_{B}(\beta)(N) \pm \bar{\nabla}_{J B}(\beta)(J N)\right)\|A\|^{2} \\
& \quad=-\left\langle\bar{R}_{A B} A+\bar{R}_{J A B} J A-\bar{R}_{A J A} J B, N\right\rangle \mp\left\langle\bar{R}_{A J B} A+\bar{R}_{J A J B} A J+\bar{R}_{A J A} B, J N\right\rangle .
\end{aligned}
$$

We omit the proof.

We conclude this section by considering the class $\mathscr{A} \mathscr{K}$.

TheOrem (6.16). Suppose $P \in \mathscr{P}$ and $J \in \mathscr{A} \mathscr{K}$. Then $M$ is a minimal variety of $\bar{M}$.

Proof. Let $N$ be a unit normal on an open subset of $M$. There exists an orthogonal frame field $\left\{E_{1}, J E_{1}, E_{2}, J E_{2}, E_{3}, J E_{3}\right\}$ on this open subset such that $\left\|E_{\imath}\right\|^{2}$ $= \pm 1$ and $P\left(N, E_{1}, E_{2}\right)=\left\|E_{3}\right\|^{2} E_{3}$. The last equation is to be permuted cyclically. In the following computation we let $\{i, j, k\}$ be a cyclic permutation of $\{1,2,3\}$. Then

$$
\begin{aligned}
\langle H, J N\rangle= & \sum_{i=1}^{3}\left\|E_{i}\right\|^{2}\left\langle T_{E_{i}} E_{i}+T_{J E_{i}} J E_{i}, J N\right\rangle \\
= & \pm \sum\left\langle P\left(N, J T_{E_{i}} N+T_{J E_{i}} N, E_{j}\right), E_{k}\right\rangle \\
& -\sum\left\{\nabla_{E_{i}}(F)\left(E_{j}, E_{k}\right)-\nabla_{J E_{i}}(F)\left(J E_{j}, E_{k}\right)\right\},
\end{aligned}
$$

because of (6.12). Since $J \in \mathscr{Q} \mathscr{K},(6.29)$ becomes

$$
\begin{aligned}
\langle H, J N\rangle & = \pm \sum\left\|E_{i}\right\|^{2}\left\langle E_{i}, J T_{E_{i}} N+T_{J E_{i}} N\right\rangle-2 \sum \nabla_{E_{i}}(F)\left(E_{j}, E_{k}\right) \\
& =-2 d F\left(E_{1}, E_{2}, E_{3}\right) .
\end{aligned}
$$

Hence the theorem follows.

7. Some examples of six-dimensional almost complex manifolds. In this section we consider some interesting special cases of the almost complex manifolds considered in the previous section. First we note the following theorem, in which we use the notations and conventions of $\S 6$.

TheOrem (7.1). Suppose $P \in \mathscr{P}(s, t, 3)$ where $s+t=8$. Then of the conditions below, (i) implies (ii), and (ii) implies (iii).

(i) There exists a parallel vector field $N$ on $\bar{M}$ with $\|N\|^{2}=1$ such that the restriction of $N$ to $M$ is everywhere normal to $M$.

(ii) There exists a totally geodesic 7-dimensional submanifold $M^{\prime}$ of $\bar{M}$ with 
$M \subset M^{\prime}$ and a vector field $N$ on $\bar{M}$ satisfying $\|N\|^{2}=1$ whose restriction to $M^{\prime}$ is everywhere normal to $M^{\prime} ;$ furthermore the vector cross product $P^{\prime}$ induced on $M^{\prime}$ by means of Theorem (2.6) is parallel and induces the same almost complex structure $J$ on $M$ that $P$ does.

(iii) The group of the frame bundle of $M$ can be reduced to $S U(3)$ or $S U(2,1)$.

Proof. To show that (i) implies (ii) consider the distribution $\left\{x \in M_{m} \mid\left\langle x, N_{m}\right\rangle\right.$ $=0\}$ on $\bar{M}$. Since $N$ is parallel, it is not hard to see that this distribution is integrable and that its integral submanifolds are totally geodesic. Hence $M$ lies in one of the integral manifolds, say $M^{\prime}$. It follows from (4.7) that the vector cross product $P^{\prime}$ on $M^{\prime}$ is parallel. Finally Theorem (2.6) implies that $P^{\prime}$ induces the almost complex structure $J$ on $M$.

That (ii) implies (iii) follows from Theorem (2.7).

We now give a description of the almost complex structures on 6-dimensional manifolds defined by means of 2 -fold vector cross products. For the case that $M^{\prime}$ is Euclidean space and the metric on $M^{\prime}$ is the usual positive definite one, this has been done by Calabi [6], Gray [11], and Yano and Sumitomo [22]. The classes $\mathscr{K}$, $\mathscr{N} \mathscr{K}, \mathscr{S} \mathscr{K}$, and $\mathscr{H}$ are already covered by Proposition (4.7), Theorem (4.8), Theorem (4.10), and Theorem (6.5) respectively. Hence we need only consider the classes $\mathscr{2} \mathscr{K}$ and $\mathscr{H}$.

In the rest of this section we shall assume that $M^{\prime}$ is a 7-dimensional pseudoRiemannian manifold with signature $(0,7)$ or $(4,3)$ and that $M$ is an orientable hypersurface of $M^{\prime}$. If $N$ is a globally defined unit normal to $M$ then the almost complex structure $J$ on $M$ is defined by $J A=P^{\prime}(N, A)$ for $A \in \mathfrak{X}(M)$, where $P^{\prime}$ is a vector cross product on $M^{\prime}$. As usual $T$ denotes the configuration tensor field of $M$ in $M^{\prime}$.

We may always imbed $M^{\prime}$ as a totally geodesic submanifold of an 8-dimensional pseudo-Riemannian manifold $\bar{M}$ with a vector cross product $P$ which gives rise to $P^{\prime}$ by means of Theorem (2.6). If $P^{\prime}$ is parallel, $P$ may be chosen to be parallel. It follows that all the theorems of $\S 6$ apply a fortiori to the almost complex structure determined by $P^{\prime}$.

We first consider the class $2 \mathscr{K}$.

Proposition (7.2). Suppose $P^{\prime} \in \mathscr{P}$. Then

(i) $J \in \mathscr{2} \mathscr{K}$ if and only if

$$
T_{A} A=T_{J A} J A
$$

for all $A \in \mathfrak{X}(M)$; furthermore (7.1) implies that $T_{A} J A=0$ for $A \in \mathfrak{X}(M)$;

(ii) if $J \in \mathscr{Q} \mathscr{K}$ and $A$ is an eigenvector at a point $m \in M$ of $X \rightarrow T_{X} N$, then so is $J A$; hence the eigenspaces of $X \rightarrow T_{X} N$ have even dimension.

Proof. (i) follows from Theorem (6.9) and (ii) follows from the linearization of (7.1). 
Proposition (7.3). Suppose $P^{\prime} \in \mathscr{P}$ and $J \in \mathscr{2} \mathscr{K}$. Let $\kappa$ be an eigenvalue of $X \rightarrow T_{X} N$ at a point $m \in M$. Then if $A, B \in \mathfrak{X}(M)$ and $T_{A} N=\kappa A$ at $m$, we have

$$
2(B \kappa)\|A\|^{2}=\left\langle-R_{A B}^{\prime} A-R_{J A B}^{\prime} J A+R_{A J A}^{\prime} J B, N\right\rangle,
$$

where $R_{A B}^{\prime}$ denotes the curvature operator of $M^{\prime}$.

Proof. An easy calculation shows that $(B \kappa)\|A\|^{2}=-\left\langle\nabla_{B}^{\prime}(T)_{A} A, N\right\rangle$ where $\nabla^{\prime}$ is the Riemannian connection of $M^{\prime}$. Now (7.2) follows from Theorem (6.11).

In the case that $M^{\prime}$ is Euclidean space and $P^{\prime}$ is a natural vector cross product we can completely determine all quasi-Kählerian almost complex manifolds. The following theorem sharpens a result of Yano and Sumitomo [22].

THEOREM (7.4). Let $M$ be an orientable hypersurface of $M^{\prime}=R^{7}$ with unit normal $N$ and let the almost complex structure $J$ on $M$ be determined by an ordinary vector cross product $P^{\prime}$ on $M^{\prime}$ and let $M^{\prime}$ have the usual positive definite metric. If $J \in \mathscr{Q} \mathscr{K}(0,6)$, then (up to isometry of $\left.M^{\prime}\right) M$ is an open submanifold of one of the following three manifolds:

(i) a hyperplane;

(ii) a sphere $S^{6}(r)$ of radius $r>0$;

(iii) a submanifold of the form $S^{2}(r) \times \hat{M}$ where $S^{2}(r)$ is a 2-dimensional sphere of radius $r>0$ contained in a 3-dimensional subspace of $M^{\prime}$ which is closed under vector cross products, and $\hat{M}$ is a subspace of dimension 4.

Proof. Let the eigenvalues of $X \rightarrow T_{X} N$ be $\kappa_{1}, \ldots, \kappa_{6}$. If $J \in \mathscr{Q} \mathscr{K}$, it follows from Proposition (7.2) that the $\kappa_{i}^{\prime}$ 's can be numbered so that $\kappa_{1}=\kappa_{4}, \kappa_{2}=\kappa_{5}$ and $\kappa_{3}=\kappa_{6}$. Then by Proposition (7.3) the $\kappa_{i}$ 's are constant. Cartan [7] has proved that this implies that there are at most two distinct $\kappa_{i}$ 's; furthermore one of the $\kappa_{i}$ 's is zero. It follows that $M$ is an open submanifold of one of the spaces listed above, or possibly an open submanifold of $S^{4}(r) \times R^{2}$ where $S^{4}(r)$ is a 4-dimensional sphere or of $S^{2}(r) \times R^{4}$ where $S^{2}(r)$ is not contained in a 3-dimensional subspace of $M^{\prime}$ closed under vector cross products. To complete the proof we exclude the latter two possibilities.

We prove that the almost complex structure $J$ on $S^{4}(r) \times R^{2}$ cannot be quasiKählerian; the proof in the other case is similar. Now, no 5-dimensional subspace of $M^{\prime}$ can be closed under 2-fold vector cross products. It follows that there exists $A \in \mathfrak{X}\left(S^{4}(r) \times R^{2}\right)$ which is tangent to $S^{4}(r)$ such that $J A$ is not tangent to $S^{4}(r)$. Then the configuration tensor $T$ of $S^{4}(r) \times R^{2}$ is given by the formula

$$
T_{A} B=-\left(1 / r^{2}\right)\left\langle A_{1}, B_{1}\right\rangle N
$$

where $N$ is the outward normal and $A_{1}$ denotes the component of $A$ tangent to $S^{4}(r)$. If $A$ is chosen as above then from (7.3) it follows that $T_{A} A \neq T_{J A} J A$ and so $J \notin \mathscr{Q} \mathscr{K}$.

We note two further formulas for the class $\mathscr{2} \mathscr{K}$. 
Proposition (7.5). Suppose $P^{\prime} \in \mathscr{P}$ and $J \in \mathscr{Q} \mathscr{K}$. Then for $A, B, C, D \in \mathfrak{X}(M)$ we have

$$
\left\langle R_{A B} C, D\right\rangle-\left\langle R_{J A J B} J C, J D\right\rangle=\left\langle R_{A B}^{\prime} C, D\right\rangle-\left\langle R_{J A J B}^{\prime} J C, J D\right\rangle .
$$

Furthermore if $M^{\prime}$ is flat, then

$$
k(J A, J B)=k(A, B)=\left\langle T_{A} B, H\right\rangle-\gamma(A, J B) .
$$

Proof. The Gauss equation [10] implies (7.4), and (7.5) follows from (7.4).

Next we turn to the class $\mathscr{A} \mathscr{K}$ and give a short proof of a result due to Yano and Sumitomo [22] analogous to Theorem (4.9). However, the theorem we are about to prove is deeper because it uses Theorem (7.4), but more restricted since it only works for positive definite metric.

THeOREM (7.6). Assume that the hypotheses of Theorem (7.4) are satisfied. If in addition $J \in \mathscr{A} \mathscr{K}(0,6)$, then $M$ is an open submanifold of a hyperplane of $R^{7}$.

Proof. $M$ is an open submanifold of one of the three manifolds listed in Theorem (7.4). On the other hand, by Theorem (6.16) $M$ is a minimal variety of $R^{7}$. Hence $M$ must be an open submanifold of a hyperplane.

We remark that in the general case where $M^{\prime}$ is not Euclidean space $R^{7}$, a result slightly stronger than Theorem (6.16) holds.

TheOREM (7.7). Suppose $P^{\prime} \in \mathscr{A} \mathscr{P}(s, t, 2)$ where $s+t=7$ and $J \in \mathscr{A} \mathscr{K}(s, t-1)$. Then $M$ is a minimal variety of $M^{\prime}$.

The proof is given in [11] (for the positive definite case).

Next we turn to the explicit construction of nonflat 6-dimensional Kähler manifolds defined by means of 3-fold vector cross products. This is accomplished in the next theorem, in which a very strange phenomenon occurs: The property of being Kählerian depends upon the proper choice of 3-fold vector cross product.

Let $\bar{M}=R^{8}$ have either signature $(0,8)$ or $(4,4)$, let $\tilde{M}$ be a 4 -dimensional subspace closed under a given 3 -fold vector cross product $P$ on $\bar{M}$, and assume that the induced metric on $\tilde{M}$ has signature $(0,4)$ or $(2,2)$. Choose any linear almost complex structure $\tilde{J}: \tilde{M} \rightarrow \tilde{M}$ and denote the induced manifold almost complex structure on $\tilde{M}$ by $\tilde{J}$ also. Let $M^{*}$ be any 2-dimensional submanifold of $\tilde{M}$. Write $\bar{M}=\tilde{M} \oplus \hat{M}$ and choose unit normal vector fields $N$ and $J N$ locally on $M^{*} \times \hat{M}$ so that $N \wedge J N$ is globally defined on $M^{*} \times \hat{M}$. As usual we denote by $J$ the almost complex structure on $M=M^{*} \times \tilde{M}$ defined by $J A=P(N, J N, A)$ for $A \in \mathfrak{X}\left(M^{*} \times \hat{M}\right)$, where $P$ is an ordinary vector cross product on $R^{8}$.

THEOREM (7.8). Suppose that $M^{*}$ is an almost complex submanifold of $\tilde{M}$ with respect to $\tilde{J}$. Hence up to sign $\tilde{J}$ and $J$ agree on $\mathfrak{X}\left(M^{*}\right)$ and $\mathfrak{X}\left(M^{*}\right)^{\perp} \cap \mathfrak{X}(\tilde{M})$. Then

(i) the almost complex structure $J$ on $M^{*} \times \hat{M}$ is Hermitian;

(ii) if $P$ satisfies (2.3), then $J$ is Kählerian if and only if (a) $J= \pm \tilde{J}$ on $\mathfrak{X}\left(M^{*}\right)$ $\cup\left(\mathfrak{X}\left(M^{*}\right)^{\perp} \cap \mathfrak{X}(\tilde{M})\right)$ or (b) $M^{*}$ is totally geodesic in $M$; 
(iii) if $P$ satisfies (2.4), then $J$ is Kählerian if and only if (a) $J= \pm \tilde{J}$ on $\mathfrak{X}\left(M^{*}\right)$ and $J=\mp \tilde{J}$ on $\mathfrak{X}\left(M^{*}\right)^{\perp} \cap \mathfrak{X}(\tilde{M})$, or (b) $M^{*}$ is totally geodesic in $\tilde{M}$.

Proof. Since $M^{*}$ is an almost complex submanifold of $\tilde{M}$, it is a minimal subvariety of $M[10]$. Hence $M^{*} \times \hat{M}$ is a minimal variety of $\bar{M}$, and in fact if $A \in \mathfrak{X}\left(M^{*} \times \hat{M}\right)$ with $\|A\|^{2} \neq 0$, then $\|A\|^{-2}\left(T_{A} A+T_{J A} J A\right)$ is the mean curvature vector of $M^{*} \times \hat{M}$. Hence (i) follows.

We prove (ii); the proof of (iii) is similar. For $A, B \in \mathfrak{X}\left(M^{*} \times \hat{M}\right)$ we let $A_{1}$ and $B_{1}$ denote the components of $A$ and $B$ tangent to $M^{*}$. If $P$ is given by (2.3) then

$$
0=T_{A} J B-J T_{A} B=T_{A_{1}} J B_{1}-J T_{A_{1}} B_{1} .
$$

Hence either (ii)(a) holds, or $2 J T_{A_{1}} B_{1}=0$ for all $A, B \in \mathfrak{X}\left(M^{*} \times \hat{M}\right)$. In the latter case $M^{*}$ must be flat.

Finally we consider the case when $M^{*}$ is not necessarily an almost complex submanifold of $\tilde{M}$.

THEOREM (7.9). Let $J$ denote the almost complex structure on $M^{*} \times \hat{M}$ defined by $J A=P(N, J N, A)$ for $A \in \mathfrak{X}\left(M^{*} \times \hat{M}\right)$. Then

(i) $J \in \mathscr{H}$ if and only if $M^{*}$ is a minimal variety of $M$;

(ii) $J \in \mathscr{Q} \mathscr{K}, J \notin \mathscr{A} \mathscr{K} \cup \mathscr{N} \mathscr{K}$ if and only if $M^{*}$ is locally isometric to a sphere $S^{2}(r)$, imbedded in the usual way in $\tilde{M}$;

(iii) $M \in \mathscr{S} \mathscr{K}, M \notin \mathscr{Q} \mathscr{K} \cap \mathscr{H}$ if and only if $M^{*}$ is neither a minimal variety nor locally isometric to a sphere $S^{2}(r)$, imbedded in the usual way in $\tilde{M}$.

Proof. (i) and (ii) may be proved in the same fashion as (i) of Theorem (7.8). Then (iii) follows from (i) and (ii).

\section{BIBLIOGRAPHY}

1. M. Berger, Sur les groupes d'holonomie homogène des variétés à connexion affine et des variétés riemanniennes, Bull. Soc. Math. France 83 (1955), 279-330.

2. E. Bonan, Sur des variétés riemanniennes à groupe d'holonomie $G_{2}$ ou Spin (7), C. R. Acad. Sci. Paris 262 (1966), 127-129.

3. A. Borel, La cohomologie mod 2 de certains espaces homogènes, Comment. Math. Helv. 27 (1953), 165-197.

4. - Sur l'homologie et la cohomologie des groupes de Lie compacts connexes, Amer. J. Math. 76 (1954), 273-342.

5. R. Brown and A. Gray, Vector cross products, Comment. Math. Helv. 42 (1967), 222-236.

6. E. Calabi, Construction and properties of some 6-dimensional almost complex manifolds, Trans. Amer. Math. Soc. 87 (1958), 407-438.

7. É. Cartan, Sur quelques familles remarquables d'hypersurfaces, C. R. Math. Liege (1939), $30-41$.

8. P. Dombrowski, On the geometry of the tangent bundle, J. Reine Angew. Math. 210 (1962), 73-88.

9. B. Eckmann, Stetige Lösungen linearer Gleichungssysteme, Comment. Math. Helv. 15 (1943), 318-339.

10. A. Gray, Minimal varieties and almost Hermitian submanifolds, Michigan Math. J. 12 (1965), 273-287. 
11. A. Gyay, Some examples of almost Hermitian manifolds, Illinois J. Math. 10 (1966), 353-366.

12. - Pseudo-Riemannian almost product manifolds and submersions, J. Math. Mech. 16 (1967), 715-738.

13. S. Kotō, Some theorems on almost Kählerian spaces, J. Math. Soc. Japan 12 (1960), 422-433.

14. W. Massey, Obstructions to the existence of almost complex structures, Bull. Amer. Math. Soc. 66 (1960), 559-564.

15. B. O'Neill, Immersion of manifolds of nonpositive curvature, Proc. Amer. Math. Soc. 11 (1960), 132-134.

16. N. Steenrod, The topology of fiber bundles, Mathematical Series, Vol. 14, Princeton Univ. Press, Princeton, N. J., 1951.

17. S. Tachibana, Note on conformally flat almost-Kählerian spaces, Nat. Sci. Rep. Ochanomizu Univ. 10 (1958), 41-43.

18. E. Thomas, On the cohomology of the classifying space for the stable spinor group, Bol. Soc. Mat. Mexicana 7 (1962), 57-69.

19. H. Toda, Y. Saito and I. Yokota, Note on the generator of $\pi_{7}(\mathrm{SO}(n))$, Mem. Coll. Sci. Univ. Kyoto Ser. A 30 (1957), 227-230.

20. G. Whitehead, Note on cross-sections in Stiefel manifolds, Comment Math. Helv. 37 (1962), 239-240.

21. J. Wolf, Spaces of constant curvature, McGraw-Hill, New York, 1967.

22. K. Yano and T. Sumitomo, Differential geometry of hypersurfaces in a Cayley space, Proc. Roy. Soc. Edinburgh Sect. A 66 (1962/64), 216-231.

UNIVERSITY OF MARYLAND, College Park, Maryland 